\title{
Roper resonances and quasi-normal modes of Skyrmions
}

\author{
C. Adam, ${ }^{a}$ M. Haberichter, ${ }^{b, c}$ T. Romanczukiewicz ${ }^{d}$ and A. Wereszczynski ${ }^{d}$ \\ ${ }^{a}$ Departamento de Física de Partículas and Instituto Galego de Física de Altas Enerxias (IGFAE), \\ Universidad de Santiago de Compostela, \\ E-15782 Santiago de Compostela, Spain \\ ${ }^{b}$ Institut für Physik, Universität Oldenburg, \\ Postfach 2503, D-26111 Oldenburg, Germany \\ ${ }^{c}$ Department of Mathematics and Statistics, University of Massachusetts, \\ Amherst, Massachusetts 01003-4515, U.S.A. \\ ${ }^{d}$ Institute of Physics, Jagiellonian University, \\ Lojasiewicza 11, Kraków, Poland \\ E-mail: adam@fpaxp1.usc.es, mhaberichter@gmail.com, \\ trom@th.if.uj.edu.pl, andwereszczynski@gmail.com
}

ABSTRACT: Radial vibrations of charge one hedgehog Skyrmions in the full Skyrme model are analysed. We investigate how the properties of the lowest resonance modes (quasi normal modes) - their frequencies and widths - depend on the form of the potential (value of the pion mass as well as the addition of further potentials) and on the inclusion of the sextic term. Then we consider the inverse problem, where certain values for the frequencies and widths are imposed, and the field theoretic Skyrme model potential giving rise to them is reconstructed. This latter method allows to reproduce the physical Roper resonances, as well as further physical properties of nucleons, with high precision.

KEywords: Chiral Lagrangians, Effective Field Theories, Solitons Monopoles and Instantons

ARXiv EPrint: 1710.00837 


\section{Contents}

1 Introduction 1

2 Radial vibrations of charge one Skyrmions 4

2.1 Hedgehog ansatz 4

2.2 Linear perturbation 5

2.3 Finding resonances 6

2.4 The full time evolution $\quad 7$

3 The standard Skyrme model and the role of the potential 8

3.1 The pion mass potential 8

$\begin{array}{ll}3.2 & \text { Deformed pion mass potential } \\ 3.30\end{array}$

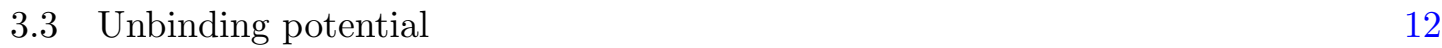

$\begin{array}{lll}3.4 & \text { Time dependence } & 13\end{array}$

4 The full Skyrme model and the role of the sextic term 15

5 Reconstruction $\quad \mathbf{2 0}$

$5.11+1$ dimensional example 20

5.2 Skyrme model from the effective potential 22

$\begin{array}{lll}5.3 & \text { Roper reconstruction details } & 22\end{array}$

$\begin{array}{llr}6 & \text { Summary } & 27\end{array}$

\section{Introduction}

The Skyrme model [1-3] is an effective field theory (EFT) which bridges the underlying fundamental theory, Quantum Chromodynamics (QCD) - well understood in the perturbative, high energy regime - with the non-perturbative low energy region, beyond a scale where confinement and hadronisation leave only colorless states as observable particles. The natural field degrees of freedom in this regime are the lightest quasi-particles, i.e., pions. The main attractiveness of the model results from the fact that this field content is sufficient to describe, in principle, all other excitations - baryons and atomic nuclei which emerge as non-perturbative states in such a mesonic fluid, or in the modern language, as topological solitons.

This solitonic framework received further support from the large $N_{c}$ limit where it has been rigorously shown that QCD can be described by a weakly interacting theory of mesons [4-6]. Moreover, the pertinent topological index of the Skyrme model has been identified with the baryon charge. Finally, after the semiclassical quantization of zero 
modes of the classical solutions of the Skyrme model (Skyrmions) in a given topological sector (baryon charge) one got access to fermionic excitations of this classically purely bosonic theory. This opened the way for a realistic application of the Skyrme model for the description of baryons [7-14] (proton, neutron, $\Delta$ resonances), lighter nuclei and their excitation bands [15-23] as well as higher nuclei, binding energies [24-30] and even infinite nuclear matter which defines properties of neutron stars [31-34].

In the baryon number one sector and with the $\mathrm{SU}(2)$ flavor group, there are two simple types of degrees of freedom of nucleons whose excitations can lead to new quasiparticles. First of all, an (iso)rotational excitation explains the $\Delta$ resonance. Another possibility is to excite some vibrational degrees of freedom. This leads to new states which carry the same spin and isospin quantum numbers as the nucleons, i.e., the Roper resonances. The first three reasonably well established Ropers on top of the nucleons are: $N(1440), N(1710)$, $N(1880)$, which are highly short-lived quasi-particles. Specifically, the first Roper $N(1440)$ has a relatively wide Breit-Wigner width $\Gamma \simeq 300 \mathrm{MeV}$ (and quite short mean life time $\tau=\hbar / \Gamma$ ), while for the next two we have $\Gamma \simeq 250 \mathrm{MeV}$ (although one should be aware of some uncertainties) [35].

The first step towards understanding the Roper resonances within the Skyrme framework is to carefully study the existence and properties of resonance modes (quasi-normal modes) in the classical model. Let us remark that quasi-normal modes also play an important role for soliton scattering (see, e.g., [36]), although this issue is beyond the scope of the present paper.

The most general Poincaré invariant Skyrme model with a standard Hamiltonian formulation reads

$$
\mathcal{L}=\mathcal{L}_{0}+\mathcal{L}_{2}+\mathcal{L}_{4}+\mathcal{L}_{6},
$$

consisting of four different terms which possess different mathematical properties and can be related to distinct phenomenological features. The first is a potential (non-derivative) term $\mathcal{L}_{0}=-\mathcal{U}(U)$ where $U$ is the $\mathrm{SU}(2)$-valued Skyrme field. This is the only term which is not completely fixed. It must provide a proper mass for the perturbative pionic fields but is otherwise quite arbitrary. We shall, however, always assume that $\mathcal{U}$ only depends on $\operatorname{Tr} U$, such that the isospin symmetry remains unbroken. The importance of different potential terms for nuclear binding energies has been understood quite recently [24-30]. Secondly, we have the usual sigma-model term which is just a kinetic term for the fields

$$
\mathcal{L}_{2}=-\lambda_{2} L_{2}=-\lambda_{2} \frac{1}{2} \operatorname{Tr}\left(L_{\mu} L^{\mu}\right),
$$

where $L_{\mu} \equiv U^{\dagger} \partial_{\mu} U$. Thirdly, there is a four-derivative part, the so-called Skyrme term

$$
\mathcal{L}_{4}=\lambda_{4} L_{4}=\lambda_{4} \frac{1}{4} \operatorname{Tr}\left(\left[L_{\mu}, L_{\nu}\right]^{2}\right),
$$

which was originally introduced to circumvent the Derrick argument for the non-existence of static solitons, and therefore was mandatory for the model [1-3]. Physically, this term contributes to two-particle repulsive interactions. Finally there is a six derivative term

$$
\mathcal{L}_{6}=\lambda_{6} L_{6}=-\lambda_{6}\left(24 \pi^{2}\right)^{2} \mathcal{B}_{\mu} \mathcal{B}^{\mu}, \quad \mathcal{B}^{\mu}=\frac{1}{24 \pi^{2}} \epsilon^{\mu \nu \rho \sigma} \operatorname{Tr} L_{\nu} L_{\rho} L_{\sigma},
$$


where $\mathcal{B}_{\mu}$ is the topological (baryon) current. By construction this term, being a topological current squared, describes some coherent, multi-particle interactions. It is the leading term of the model in the high energy density (baryon density) limit, providing the main contribution to the mean-field equation of state in this regime [37]. This can happen inside (bulk) of atomic nuclei but also in cold dense nuclear matter (at high pressure and/or density $[38,39])$. Phenomenologically, this term can also be related to a repulsive interaction mediated by the $\omega$ pseudo-vector meson [38-40].

Before we proceed to the main part of the paper, we briefly review the current knowledge on vibrational modes in the Skyrme model framework and the possible relation to Ropers. The simplest approach, that is the collective mode approximation, has been applied to the massless Skyrme model $\mathcal{L}_{2}+\mathcal{L}_{4}$ [41] and to the sextic extension $\mathcal{L}_{2}+\mathcal{L}_{4}+\mathcal{L}_{6}$ [42]. This has been further developed (by coupling the vibrational excitations to the rotational modes) which resulted in a derivation of the Roper states for nucleons as well as for the $\Delta$ resonance [43-45]. Linear perturbation theory has also been applied [42, 46, 47], where the main finding was the nonexistence of an oscillating mode unless a very heavy pion is considered [47]. This result was obtained for the usual Skyrme potential and does not have to be true for other potentials [48]. In any case, it is expected that Ropers should rather be described by resonance modes [46, 48]. Such a resonance has, in fact, been found in the potential-less Skyrme model $\mathcal{L}_{2}+\mathcal{L}_{4}$ [49], however, no relation to the Ropers or influence of the pion mass has been discussed.

It is the main aim of the present work to fill the gap and carefully analyse the properties of the first (three) lowest resonance modes in the full Skyrme model. Especially, we want to perform our investigation for two types of generalisations of the Skyrme model which are known to cure the classical binding energy problem of the original proposal.

1. First of all, we want to systematically study how a specific form of the potential influences the properties of resonance modes (frequency and width). This will be done for potentials considered by Lin and Piette [48] and for an unbinding potential i.e., a potential which is known to reduce the unphysical large binding energies of Skyrmions. Here we will also study the impact of the value of the pion mass.

2. Secondly, we will investigate these questions in the Skyrme model where the sextic term is taken into account.

3. Finally, we shall study the inverse problem, starting from a Schrödinger equation (Sturm-Liouville problem) which leads to certain low-lying resonance modes close to the Roper resonances. We then reconstruct the corresponding Skyrme model (i.e., the potential).

The paper is organized as follows. In section 2, the radial vibrations of the hedgehog Skyrmion are analyzed. We formulate the corresponding Sturm-Liouville problem with an algebraic effective potential (in terms of the unperturbed solution). Section 3 is devoted to the investigation of the impact of different forms of the potential. In section 4 we take into account the sextic term. The inverse problem (reconstruction) is considered in section 5 . In the last section we summarize our results. 


\section{Radial vibrations of charge one Skyrmions}

\subsection{Hedgehog ansatz}

We consider the general Skyrme model $\mathcal{L}_{0246}=\mathcal{L}_{0}+\mathcal{L}_{2}+\mathcal{L}_{4}+\mathcal{L}_{6}$. It is convenient to choose the following parameterization of the coupling constants

$$
\lambda_{2}=\frac{f_{\pi}^{2}}{8}, \quad \lambda_{4}=\frac{1}{8 e^{2}}, \quad \lambda_{0}=\frac{f_{\pi}^{2}}{8} m_{\pi}^{2}, \quad \lambda_{6}=\frac{2}{(24)^{2} e^{4} f_{\pi}^{2}} \epsilon^{2} .
$$

Then, we introduce the physical energy and length scales $\mathcal{E}=f_{\pi} / 4 e$ and $\ell=2 / e f_{\pi}$. This leads to the following Lagrangian in Skyrme units

$$
L=\frac{f_{\pi}}{4 e} \int d^{3} x\left(\frac{1}{2} \operatorname{Tr} L_{\mu} L^{\mu}-\frac{1}{16} \operatorname{Tr}\left[L_{\mu}, L_{\nu}\right]\left[L^{\mu}, L^{\nu}\right]-\epsilon^{2} \pi^{4} \mathcal{B}_{\mu}^{2}-m^{2} \mathcal{U}\right)
$$

where $m=2 m_{\pi} /\left(f_{\pi} e\right)$ is the pion mass in Skyrme units. The multiplicative factor in front of the integral provides a transition from Skyrme units to physical units. In the usual parametrization $U=e^{i \xi \vec{n} \cdot \vec{\tau}}$, where $\xi$ is a real scalar and $\vec{n}$ the unit three component vector field. Here $\vec{\tau}$ are the Pauli matrices. Next, we assume the stereographic projection

$$
\vec{n}=\frac{1}{1+|u|^{2}}\left((u+\bar{u}),-i(u-\bar{u}), 1-|u|^{2}\right)
$$

where $u$ is a complex field, $\bar{u}$ is its complex conjugate and $|u|^{2} \equiv u \bar{u}$. Then the derivative terms take the following form

$$
\begin{aligned}
\frac{1}{2} \operatorname{Tr} L_{\mu} L^{\mu} & =\xi_{\mu} \xi^{\mu}+4 \sin ^{2} \xi \frac{u_{\mu} \bar{u}^{\mu}}{\left(1+|u|^{2}\right)^{2}}, \\
\frac{1}{4} \operatorname{Tr}\left[L_{\mu}, L_{\nu}\right]\left[L^{\mu}, L^{\nu}\right] & =16 \sin ^{2} \xi\left(\xi_{\mu} \xi^{\mu} \frac{u_{\mu} \bar{u}^{\mu}}{\left(1+|u|^{2}\right)^{2}}-\frac{\xi_{\mu} \bar{u}^{\mu} \xi_{\nu} u^{\nu}}{\left(1+|u|^{2}\right)^{2}}\right)+16 \sin ^{4} \xi \frac{\left(u_{\mu} \bar{u}^{\mu}\right)^{2}-u_{\mu} u^{\mu} \bar{u}_{\nu} \bar{u}^{\nu}}{\left(1+|u|^{2}\right)^{2}} \\
\left(\epsilon^{\mu \nu \rho \sigma} \operatorname{Tr} L_{\nu} L_{\rho} L_{\sigma}\right)^{2} & =\frac{\sin ^{4} \xi}{\left(1+|u|^{2}\right)^{4}}\left(\epsilon^{\mu \nu \rho \sigma} \xi_{\nu} u_{\rho} \bar{u}_{\sigma}\right)^{2} .
\end{aligned}
$$

The potential is assumed to be a function of the scalar field $\xi$ only.

We want to consider how the charge one Skyrmion reacts under a spherically symmetric perturbation. Such a static stable $B=1$ solution is given by the hedgehog ansatz

$$
\xi=\xi_{0}(r), \quad u=\tan \frac{\theta}{2} e^{i \phi}
$$

where the static profile $\xi_{0}$ solves the following $\mathrm{ODE}\left(\xi_{0}^{\prime} \equiv(d / d r) \xi_{0}\right)$

$$
\begin{aligned}
& 2\left(r^{2}+2 \sin ^{2} \xi_{0}+\frac{\epsilon^{2} \sin ^{4} \xi_{0}}{4 r^{2}}\right) \xi_{0}^{\prime \prime}= \\
& -4 r \xi_{0}^{\prime}-2 \sin 2 \xi_{0}\left(\xi_{0}^{\prime 2}-1-\frac{\sin ^{2} \xi_{0}}{r^{2}}\right)+m^{2} r^{2} \mathcal{U}_{\xi}+\epsilon^{2}\left(\frac{\sin ^{4} \xi_{0}}{r^{3}} \xi_{0}^{\prime}-\frac{\sin ^{2} \xi_{0} \sin 2 \xi_{0}}{2 r^{2}} \xi_{0}^{\prime 2}\right) .
\end{aligned}
$$

We assume that the initial perturbation does not break the spherical symmetry of the solution. Hence the $S^{2}$ part of the solution ( $u$ field) remains unchanged while the profile 
function is a function of the radial coordinate and time $\xi=\xi(r, t)$. This leads to the reduced Lagrangian

$$
L=\frac{f_{\pi}}{4 e} \int d^{3} x\left[\xi_{\mu} \xi^{\mu}\left(1+2 \frac{\sin ^{2} \xi}{r^{2}}+\frac{\epsilon^{2} \sin ^{4} \xi}{4 r^{4}}\right)-2 \frac{\sin ^{2} \xi}{r^{2}}\left(1+\frac{\sin ^{2} \xi}{2 r^{2}}\right)-m^{2} \mathcal{U}(\xi)\right] .
$$

This equation is the starting point for our further analysis.

\section{$2.2 \quad$ Linear perturbation}

In the linear perturbation, we consider small fluctuations around the static soliton, $\xi=\xi_{0}+\eta(r, t)$. The resulting part of the Lagrangian quadratic in $\eta$ reads $\left(\mathcal{U}^{\prime} \equiv(d / d \xi) \mathcal{U}\right)$

$$
\begin{aligned}
L_{\eta}= & \frac{f_{\pi}}{4 e} 4 \pi \int d r \eta_{\mu} \eta^{\mu}\left(r^{2}+2 \sin ^{2} \xi_{0}+\frac{\epsilon^{2} \sin ^{4} \xi_{0}}{4 r^{2}}\right)+2\left(2 \sin 2 \xi_{0}+\frac{\epsilon^{2}}{r^{2}} \sin ^{3} \xi_{0} \cos \xi_{0}\right) \xi_{0}^{\prime} \eta \eta^{\prime} \\
& +\eta^{2}\left(2 \cos 2 \xi_{0}\left(\left(\xi_{0}^{\prime}\right)^{2}-1\right)-\frac{1}{r^{2}}\left(\sin ^{2} 2 \xi_{0}+2 \cos 2 \xi_{0} \sin ^{2} \xi_{0}\right)+\frac{\epsilon^{2}}{4 r^{2}}\left(\xi_{0}^{\prime}\right)^{2}\left(6 \sin ^{2} \xi_{0}-8 \sin ^{4} \xi_{0}\right)\right. \\
& \left.-\frac{m^{2}}{2} r^{2} \mathcal{U}^{\prime \prime}\right) .
\end{aligned}
$$

For the oscillation (resonance) spectrum we assume $\eta(t, r)=e^{i \omega t} \eta(r)$ with $\omega=\Omega+i \Gamma$. Then, we arrive at the following functions for the corresponding Sturm-Liouville problem in its canonical form $\left(p \eta^{\prime}\right)^{\prime}+q \eta=\omega^{2} s \eta$ (for details of the procedure, see appendix A of [50]),

$$
p(r)=s(r)=\left(r^{2}+2 \sin ^{2} \xi_{0}+\frac{\epsilon^{2} \sin ^{4} \xi_{0}}{4 r^{2}}\right)
$$

and

$$
\begin{aligned}
q(r)= & \xi_{0}^{\prime \prime}\left(-2 \sin 2 \xi_{0}-\frac{\epsilon^{2}}{2 r^{2}} \sin ^{2} \xi_{0} \sin 2 \xi_{0}\right)+\left(\xi_{0}^{\prime}\right)^{2}\left(-2 \cos 2 \xi_{0}-\frac{\epsilon^{2}}{2 r^{2}}\left(3 \sin ^{2} \xi_{0}-4 \sin ^{4} \xi_{0}\right)\right) \\
& +\xi_{0}^{\prime} \frac{\epsilon^{2}}{r^{3}} \sin ^{2} \xi_{0} \sin 2 \xi_{0}+2 \cos 2 \xi_{0}+\frac{1}{r^{2}}\left(\sin ^{2} 2 \xi_{0}+2 \cos 2 \xi_{0} \sin ^{2} \xi_{0}\right)+\frac{m^{2}}{2} r^{2} \mathcal{U}_{\xi \xi}
\end{aligned}
$$

where $\xi_{0}$ is the static solution. Next, the Sturm-Liouville problem can be brought in its normal form $-u^{\prime \prime}+Q u=\omega^{2} u$, i.e., in the form of a one-dimensional Schrödinger equation with an effective (quantum mechanical) potential $Q$. In our case, we can find an exact (algebraic) expression for this effective potential. It contains two algebraic terms

$$
Q=Q_{0}+Q_{\mathcal{U}}
$$

a derivative contribution (which has been known, but only in a form which contained first and second derivatives of the field [42])

$$
Q_{0}=\frac{2}{r^{2}}-\frac{4 \sin ^{2} \xi_{0}}{\left(r^{2}+2 \sin ^{2} \xi_{0}+\frac{\epsilon^{2} \sin ^{4} \xi_{0}}{4 r^{2}}\right)^{2}}\left(r^{2}+3 \sin ^{2} \xi_{0}+3 \frac{\sin ^{4} \xi_{0}}{r^{2}}+\epsilon^{2} \frac{\sin ^{6} \xi_{0}}{4 r^{4}}\right)
$$


and a potential part (which is new)

$$
\begin{aligned}
Q_{\mathcal{U}}=\frac{m^{2} r^{2}}{2} \frac{1}{\left(r^{2}+2 \sin ^{2} \xi_{0}+\frac{\epsilon^{2} \sin ^{4} \xi_{0}}{4 r^{2}}\right)^{2}}[ & \left(r^{2}+2 \sin ^{2} \xi_{0}+\frac{\epsilon^{2} \sin ^{4} \xi_{0}}{4 r^{2}}\right) \mathcal{U}_{\xi \xi} \\
& \left.-\sin 2 \xi_{0}\left(1+\frac{\epsilon^{2}}{4 r^{2}} \sin ^{2} \xi_{0}\right) \mathcal{U}_{\xi}\right] .
\end{aligned}
$$

The potential contribution is the leading part in the asymptotic limit close to the vacuum. Indeed, $Q_{0}$ tends to zero as $r \rightarrow \infty$ (and $\xi_{0} \rightarrow 0$ ). In the no potential case $Q_{\mathcal{U}} \equiv 0$ there are no positive energy bound states (oscillating modes) [49]. There are also no negative energy states, which guarantees the linear stability of the $B=1$ Skyrmion. Oscillating modes can appear if a potential is taken into account. Then, asymptotically the effective potential tends to

$$
Q_{\infty} \equiv \lim _{r \rightarrow \infty} Q=\left.\frac{m^{2}}{2} \mathcal{U}_{\xi \xi}\right|_{\xi=0}
$$

which amounts to a possible appearance of (positive energy) bound states. As a consequence, a finite number of discrete modes [48] can show up. It should be stressed again that in the charge one sector the oscillating radial mode is an unwanted phenomenon, which is not supported by the existence of a corresponding particle state. On the contrary, the Roper resonances should rather be described by resonance modes. This may give some restrictions for the parameters of the model (calibration) as well as for some qualitative properties of the potential.

\subsection{Finding resonances}

Quasinormal modes are the solutions to the Schrödinger (or Sturm-Liouville) equation

$$
-u_{r r}+Q(r) u=\omega^{2} u
$$

satisfying the purely outgoing boundary condition

$$
u(r \rightarrow \infty) \sim e^{-i k r}
$$

for solutions to the wave equation of the form $\eta(r, t)=e^{i \omega t} u(r)$. This condition cannot be satisfied for real values of $k$ (because of the continuity equation which is one of the hermiticity conditions), therefore $k \in \mathbb{C}$ and hence $\omega=\sqrt{k^{2}+m^{2}} \in \mathbb{C}$. Usually, the full solution of the Schrödinger equation has both outgoing and incoming parts

$$
u(r) \approx A_{\text {in }} e^{i k r}+A_{\text {out }} e^{-i k r} .
$$

However for complex values of $k$ one of the exponents grows while the other decreases. This makes it very difficult to apply the appropriate boundary condition. We rewrite the potential generated by the Skyrmion as

$$
Q(r)=\frac{2}{r^{2}}+m^{2}+\delta Q(r),
$$


where $\delta Q(r)$ vanishes faster than $r^{-2}$ as $r \rightarrow \infty$. In fact, for massive fields, $\delta Q$ vanishes exponentially fast, which is a desired property. The solution can be rewritten as

$$
u(r)=A(r) u_{1}(r)+B(r) u_{2}(r),
$$

where

$$
u_{1}(r)=\left(1+\frac{i}{k r}\right) e^{i k r} \quad \text { and } \quad u_{2}(r)=\left(1-\frac{i}{k r}\right) e^{-i k r}
$$

are the solutions of the equation (2.17) for $\delta Q=0$ representing in and out-going waves, respectively. The decomposition (2.21) is not unique. Therefore we choose $A$ and $B$ to satisfy the following condition

$$
A^{\prime} u_{1}+B^{\prime} u_{2}=0, \quad \text { so that } \quad u^{\prime}=A u_{1}^{\prime}+B u_{2}^{\prime},
$$

as if $A$ and $B$ were constant, which is true for $r \rightarrow \infty$. Equation (2.17) takes the following form

$$
-A^{\prime} u_{1}^{\prime}-B^{\prime} u_{2}^{\prime}+\delta Q\left(A u_{1}+B u_{2}\right)=0 .
$$

The above first order equations can be written in matrix form as

$$
\frac{d}{d r}\left[\begin{array}{l}
A \\
B
\end{array}\right]=\delta Q\left[\begin{array}{cc}
u_{1} & u_{2} \\
-u_{1}^{\prime} & -u_{2}^{\prime}
\end{array}\right]^{-1}\left[\begin{array}{cc}
0 & 0 \\
u_{1} & u_{2}
\end{array}\right]\left[\begin{array}{l}
A \\
B
\end{array}\right]
$$

or in more explicit form

$$
\frac{d}{d r}\left[\begin{array}{l}
A \\
B
\end{array}\right]=\frac{i \delta Q}{2 k}\left[\begin{array}{cc}
-u_{1} u_{2} & -u_{2}^{2} \\
u_{1}^{2} & u_{1} u_{2}
\end{array}\right]\left[\begin{array}{l}
A \\
B
\end{array}\right] .
$$

The initial condition required for regular solutions at $r=0$ is $A(0)=B(0)$. Using the scalability of the linear equation we impose the condition $A(0)=B(0)=1$. With these initial conditions the singular term in the equation is cancelled.

Moreover, the above equations are much easier to solve numerically than the original eq. (2.17) since the coefficients $A$ and $B$ become constant very fast as $\delta Q$ vanishes. More precisely, the method works if

$$
\delta Q(r) e^{2|\operatorname{im} k| r} \rightarrow 0
$$

The resonance solution has no incoming part, therefore the second condition is $A(\infty)=0$.

\subsection{The full time evolution}

Our investigations of the oscillating and resonance modes in the linearised model should be verified in a numerical analysis of the full time dependent equation which follows from the reduced Lagrangian (2.9).

To compute radial vibration spectra of $B=1$ Skyrmions fully numerically, we use a finite difference leapfrog method [52]. We discretise eq. (II.25) on a uniform grid with spatial grid size $r=[0,100]$ and temporal grid size $t=[0,1000]$. We choose $2 \times 10^{4}$ spatial grid points $(\Delta r=0.005)$ and $2 \times 10^{6}$ temporal grid points $(\Delta t=0.0005)$. The spatial derivatives used are fourth order accurate and Neumann boundaries have been imposed. 
On the other hand, for the full numerical time evolution of (2.9), we create suitable initial conditions for the leapfrog time evolution code by uniformly squeezing static $B=1$ Skyrmion solutions. Static solutions for given mass parameter $m$ and given coupling constant $\epsilon$ have been obtained by solving eq. (2.8) with the collocation algorithm COLSYS $[53,54]$. To create uniformly squeezed initial conditions for our time evolution code, we rescale the radial coordinate by $r \rightarrow s r$, where the parameter $s=0.95$. For given mass $m$, the profile function $\xi_{0}(r)$ for a static Skyrmion solution has been computed on the interval $r \in[0,100]$ with $\Delta r=0.005$. We use the interpolation routine interp1d from Python's SciPy package [55] to interpolate $\xi_{0}(r)$ on $r \in[0,100]$ and to rescale $\xi_{0}(r) \rightarrow \xi_{0}(s r)$. Each squeezed initial condition is then time-evolved over $2 \times 106$ timesteps with $\Delta t=0.0005$ on a spatial grid $r \in[0,100]$ with $\Delta r=0.005$. In each time step, we measure at $r=0.1$ (inside the soliton core) the deviation from the static Skyrmion solution by recording $\xi(r=0.1, t)$.

To find the frequency components, we compute the Fast Fourier Transform (FFT) $\tilde{\xi}(r=0.1, \omega)$ of the recorded data using the MATLAB built-in function fft. For visualisation purposes, we present our results in the form of contour plots of $\log \left(\left|\tilde{\xi}_{t}(r=0.1, \omega)\right|\right) / \log (10)$.

\section{The standard Skyrme model and the role of the potential}

\subsection{The pion mass potential}

As a first example, we consider the usual pion mass potential which provides a mass for perturbative (pionic) excitations

$$
m^{2} \mathcal{U}_{\pi}=2 m^{2}(1-\cos \xi)
$$

As we already pointed out, this potential results in too high binding energies and, therefore, is not so suitable for a quantitative description of atomic nuclei. Here, we want to check its applicability to the Roper problem. For reasons of generality, we vary the mass parameter from 0 to the physically much too high value 20. Moreover, this allows us to detect resonance modes when they emerge from oscillating modes.

In figure 1 we present a scan of the Fourier transform of a radial perturbation of the hedgehog solution in the $\mathcal{L}_{024} \equiv \mathcal{L}_{0}+\mathcal{L}_{2}+\mathcal{L}_{4}$ Skyrme model with the usual potential. We vary the mass parameter from $m=0$ to $m=20$ in steps of $\Delta m=0.1$. It is clearly seen that for the (first three) oscillating modes the linear perturbation works very well (see figure 2) and there is no difference with the full computation. Some higher harmonics can easily be found. In the plot it is clearly visible that the higher harmonics $n \omega_{1}$ exist (lower right panel). The intensity (amplitude) of higher modes obviously decreases with $n$.

The main findings are the following:

1. The number of oscillating modes increases with the pion mass (figure 1). This is an obvious effect since the effective potential $Q$ forms a deeper well while $m$ grows.

2. Every resonance mode originates in a corresponding oscillating mode (figure 2). The transition happens when, while decreasing the pion mass, the frequency of the oscillating mode meets the mass threshold. Indeed, when an oscillating mode passes 


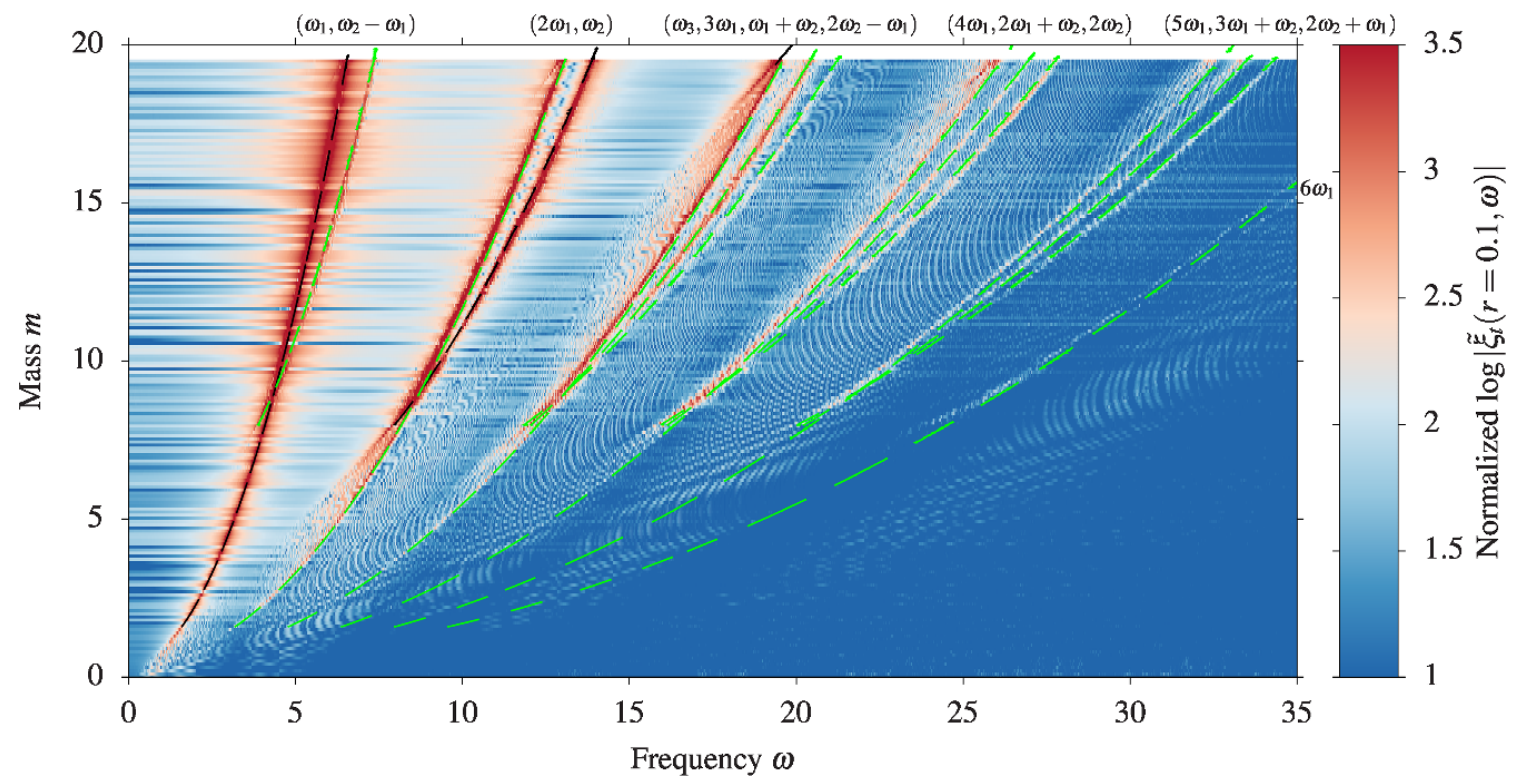

Figure 1. Power spectra of the full dynamics for the usual Skyrme potential with the pion mass $m \in[0,20]$, with the first three oscillating modes (black dashed) and higher harmonics (green dashed) denoted.
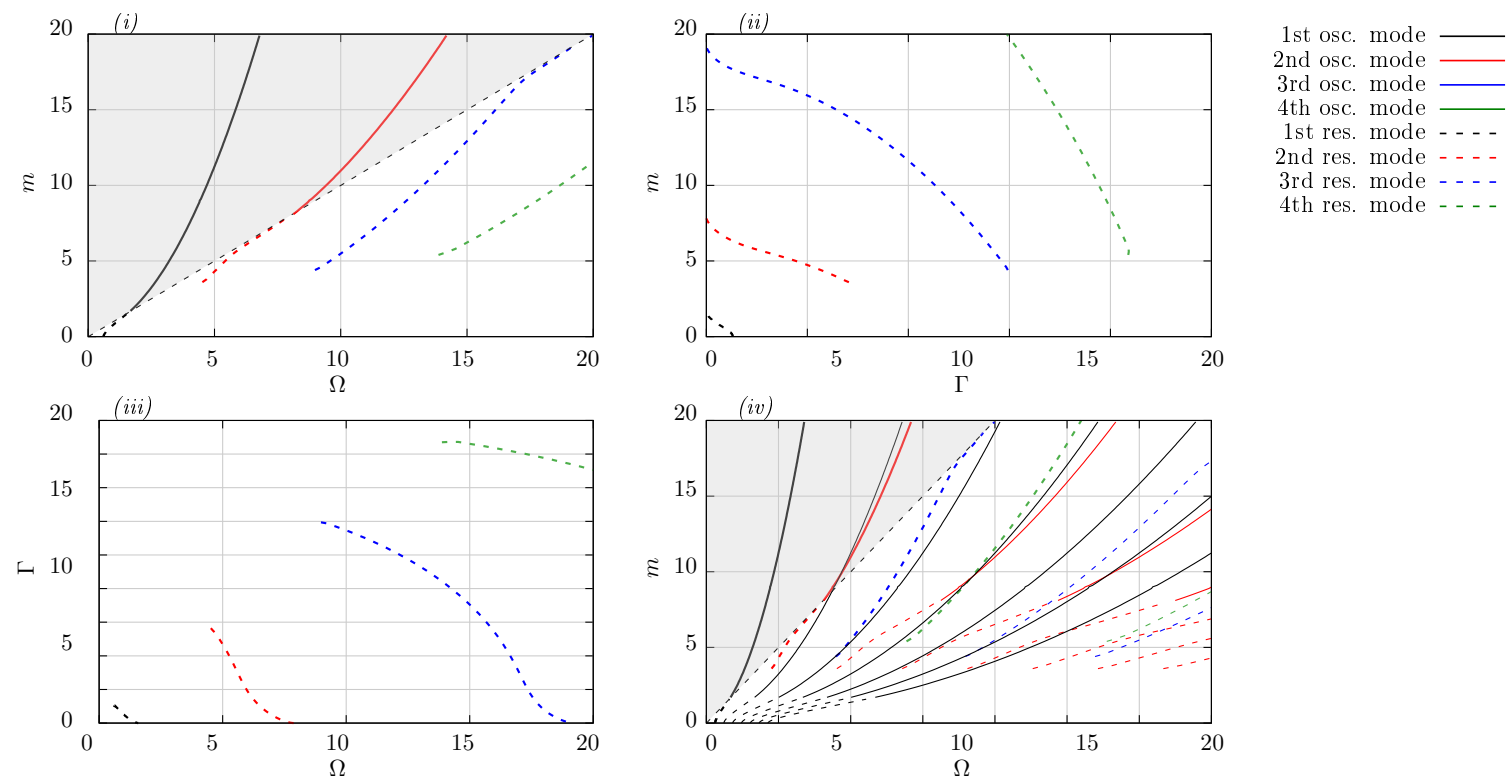

Figure 2. Resonance modes for the usual Skyrme potential with the pion mass $m \in[0,20]$. (i) Real part of the frequencies of the oscillational/resonance modes, (ii) imaginary part (damping) of the resonance modes, (iii) resonance positions in the complex plane $(\Omega, \Gamma)$, (iv) resonances/oscillational modes and their higher harmonics. 


\begin{tabular}{|c|cccc|}
\hline & $n=1$ & $n=2$ & $n=3$ & $n=4$ \\
\hline$p=1$ & 1.59 & 7.99 & 19.38 & - \\
$p=2$ & 1.18 & 6.47 & 16.22 & - \\
$p=3$ & 0.95 & 5.07 & 12.38 & - \\
$p=4$ & 0.85 & 3.98 & 9.77 & 17.56 \\
\hline
\end{tabular}

Table 1. Critical masses for the full Skyrme model with the potential $\mathcal{U}^{(p)}$. No value denotes that the critical point occurs above $m=20$.

the mass threshold line it transforms into the corresponding resonance mode. At the beginning (close to the threshold line) the mode is very narrow. It becomes wider when the mass parameter is further reduced.

3. The critical pion masses below which the $\mathrm{n}$-th oscillating mode transforms into its quasi-normal counterpart are: $m_{1}=1.59, m_{2}=7.99, m_{3}=19.38$ (see table 1 ). This means that for $m<m_{1}$ there are no oscillating modes at all, which sets an upper bound for the pion mass in this Skyrme model.

4. We control the first resonance mode in the full range of the pion mass (figure 2). Its frequency starts at $\Omega=1.59$ for $m=1.59$ and gets smaller until $\Omega=0.61$ for $m=0$ (which agrees with the original result by Bizon et al. [49]). Simultaneously, the width grows from basically 0 to $\Gamma=0.26$ for $m=0$.

5. As far as the next three resonance modes are considered, we could reduce the pion mass approximately to $m \approx 4-5$. Below this value the modes are too broad and they are beyond our accuracy. Unfortunately, this means that we are not able to reach the physical regime $m<m_{1}$. Nonetheless, we can use the values of the widths of the second, third and fourth resonance mode at the last trustable $m$ as a lower bound for the widths for physical $m$. Specifically, we found $\Gamma_{2}>1.41, \Gamma_{3}>2.98, \Gamma_{4}>4.17$. It is clearly visible that the widths of the second and higher resonance modes are much (an order of magnitude) bigger than the width of the first resonance (figure 2). This should be contrasted with the fact that the three lowest Roper states possess very similar widths. In other words, the standard Skyrme model with the pionic mass potential fails to reproduce higher Roper resonances, at least their widths.

In the next two subsections we will study how these findings change if other potentials are chosen.

\subsection{Deformed pion mass potential}

As the first example of a deformation of the standard pion mass potential we refer to Lin and Piette [48] who considered a family of multi vacuum potentials $(p=1,2,3,4)$

$$
m^{2} \mathcal{U}^{(p)}=\frac{2 m^{2}}{p^{2}}(1-\cos p \xi)
$$



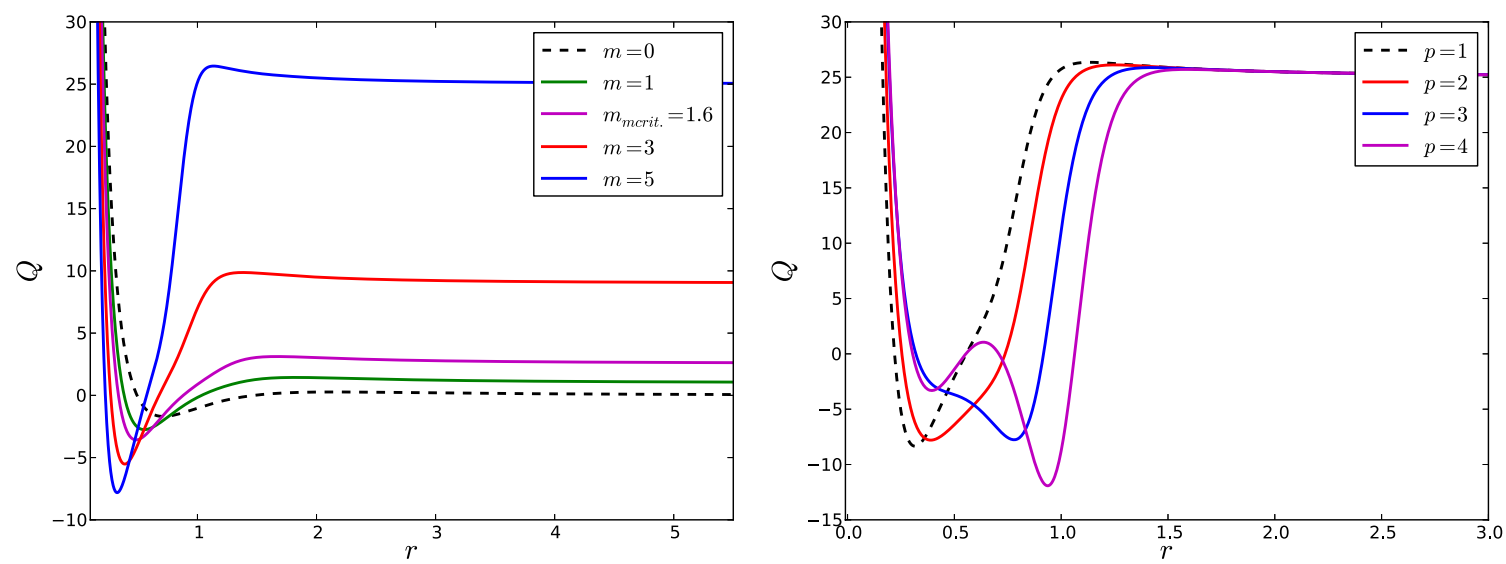

Figure 3. Effective potential $Q$ for the $L_{240}$ Skyrme model. Left: with the usual Skyrme potential and different values of the mass $m$. Right: with the Lin-Piette potentials $\mathcal{U}^{(p)}$ and $m=5$.

where $p=1$ gives the standard pion mass potential (3.1) considered above. Qualitatively, the potential $\mathcal{U}$ can influence the effective potential $Q$ (and therefore the existence and number of oscillating and resonance modes) in two simple ways. First of all, as we have already observed, increasing the pion mass leads to a higher asymptotic value of the effective potential. As a consequence, the little potential well which exists for the massless Skyrme model $\mathcal{L}_{24}$ becomes bigger (with a higher right boundary). Hence, more oscillating modes can exist. Secondly, potentials with smaller value at the anti-vacuum $(\xi=\pi)$, or in general less peaked, develop a broader well. Again, more oscillating states can emerge. Moreover, their eigen-frequencies are smaller. All this is a direct consequence of figure 3 .

This qualitative picture is fully confirmed by direct numerical computations - see figure 4. Quantitatively we found that the critical pion mass at which the first oscillating mode transforms into the lowest resonance mode decreases to $m=0.85$ for the $p=4$ potential. This implies that for this model the physical pion mass in Skyrme units cannot be larger than 0.85. In figure 5 we compare the full dynamics (power spectrum) of the $p=4$ potential with the original pion mass potential. Here $m \in[0,2]$.

Interestingly, the higher resonances now appear much faster (for much smaller pion mass) and therefore have a chance to be much narrower and even detectable in (or close to) the physical regime, i.e., when no oscillating modes exist. Indeed, in figure 6 we show the frequency and width of the lowest resonances for the $p=2$ and $p=4$ potentials. However, although the quasi-normal states are narrower, there seems to be still a significant difference between the first and higher resonances. Therefore, these potentials do not provide a reliable description of the Roper resonances, either.

The first four oscillating/resonance state for the Lin-Piette potentials with $p=1,2,3,4$ are plotted in figure 7 . Clearly, our numerical resolution allows for a precise computation for the first mode. The higher resonances require bigger accuracy and, unfortunately, they are no longer under control before the first oscillating mode turns into a resonance. 

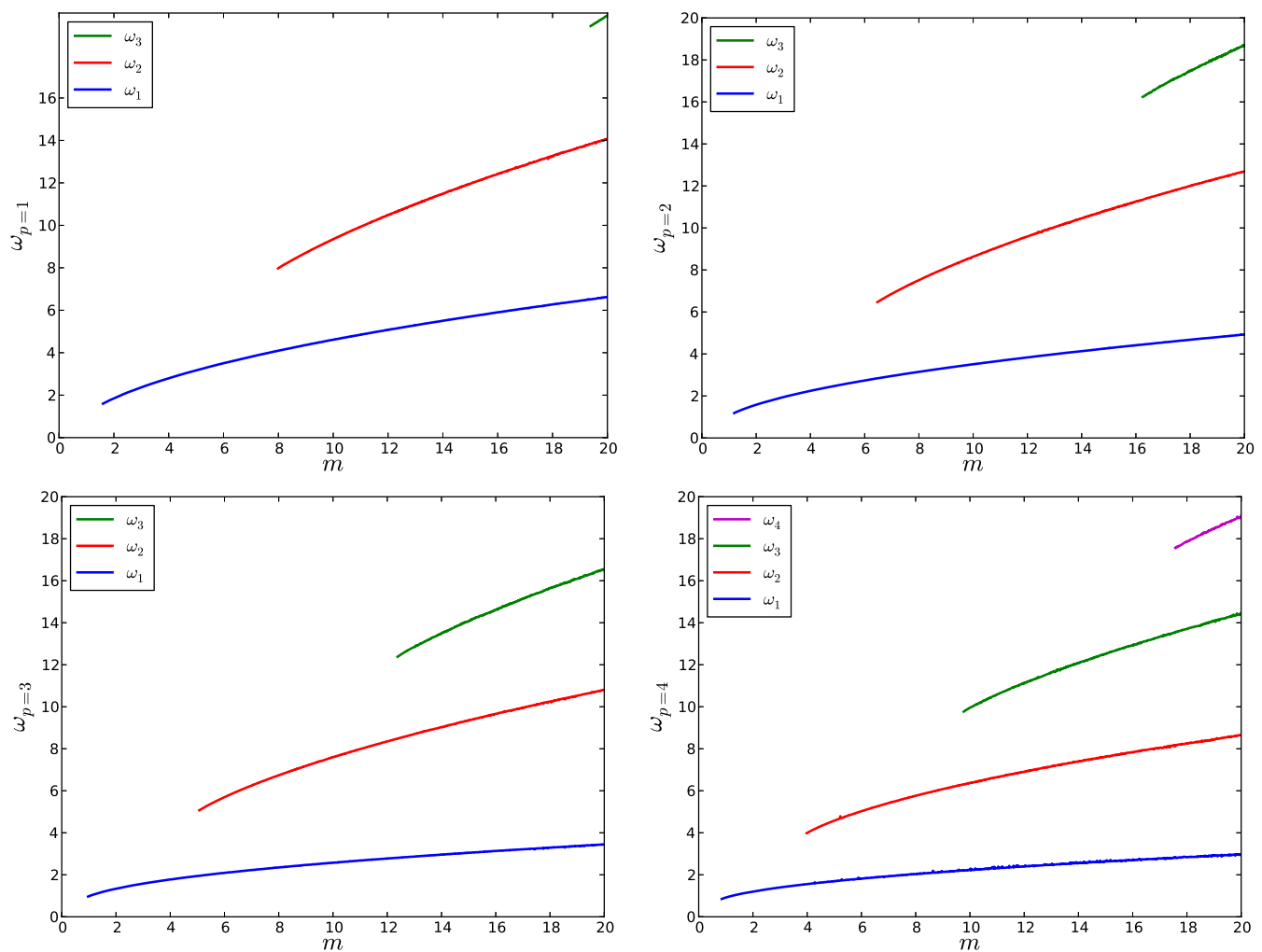

Figure 4. The frequencies of the first oscillating modes for $L_{024}$ Skyrme model as a function on the pion mass $m$ for the Lin-Piette potentials, for $p=1,2,3,4$.

\subsection{Unbinding potential}

The next type of potential is the unbinding potential considered recently $[26,27]$ (see also [28-30])

$$
m^{2} \mathcal{U}_{\alpha}=2 m^{2}(1-\cos \xi)+\frac{\alpha}{(1-\alpha)^{2}}(1-\cos \xi)^{4}
$$

where $\alpha<1$ is a parameter which controls how close to the unbinding regime we are. In the limit $\alpha=1$ (after some unit redefinition) we arrive at a Skyrme model which saturates a corresponding Bogomolny bound and does not have stable higher charge solitonic solutions. Therefore, it describes a completely unbinding model. It has been reported that physical binding energies can be obtained if $\alpha=0.95$ and

$$
f_{\pi}=36.1 \mathrm{MeV}, \quad m_{\pi}=303 \mathrm{MeV}, \quad e=3.76
$$

which correspond to $m=4.46$. Note that this is a rather big number if compared with the usual calibration scheme. In the numerical computations we found three clear resonance modes when the value of the mass parameter equals the value used for small binding energies $(m=4.46)$

$$
\begin{aligned}
& \Omega_{1}+i \Gamma_{1}=4.6077+0.1169 i \\
& \Omega_{2}+i \Gamma_{2}=8.2293+1.8342 i \\
& \Omega_{3}+i \Gamma_{3}=11.6761+4.1988 i
\end{aligned}
$$




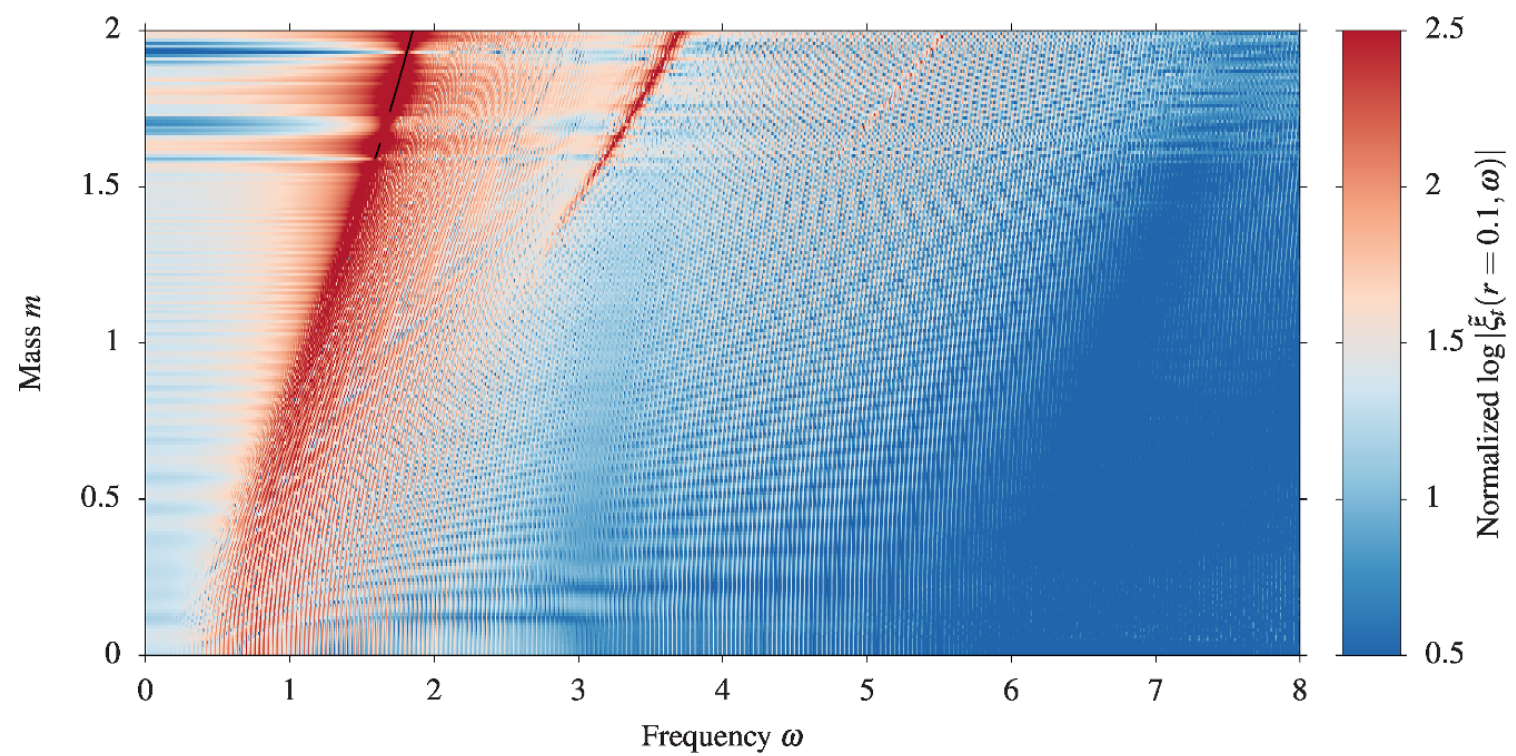

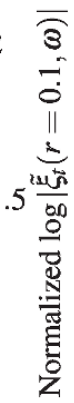

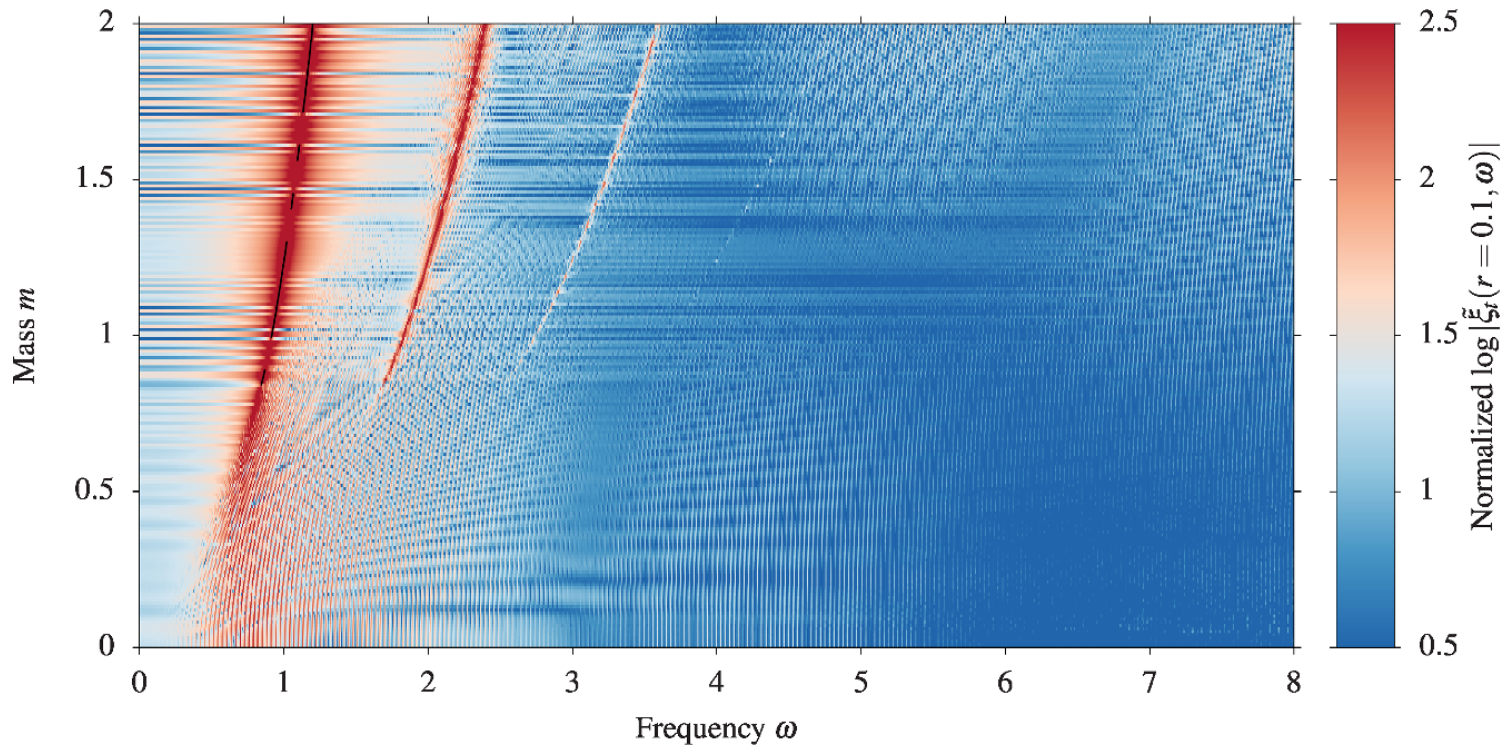

Figure 5. FFT scan of the full dynamics for the $\mathcal{L}_{024}$ Skyrme model with potential $\mathcal{U}^{(1)}$ (upper panel) and $\mathcal{U}^{(4)}$ (lower panel) with the pion mass in a physical region $m \in[0,2]$. The black dashed line denotes the first oscillating mode. Here the resolution $\delta m=0.01$.

The widths grows quite significantly. In particular, $\Gamma_{1}$ is much (more than ten times) smaller than $\Gamma_{2}$. So, for realistic parameter values the description of the Roper resonances is not satisfactory, again. Note, that for smaller values of the mass parameter the widths of the first two resonances go much closer and are, in fact, almost identical for $m$ around 2 (see figure 8).

\subsection{Time dependence}

In the linear regime, oscillational modes oscillate with constant amplitude and frequency. When nonlinear coupling is considered, the oscillational mode couples to higher harmonics. 

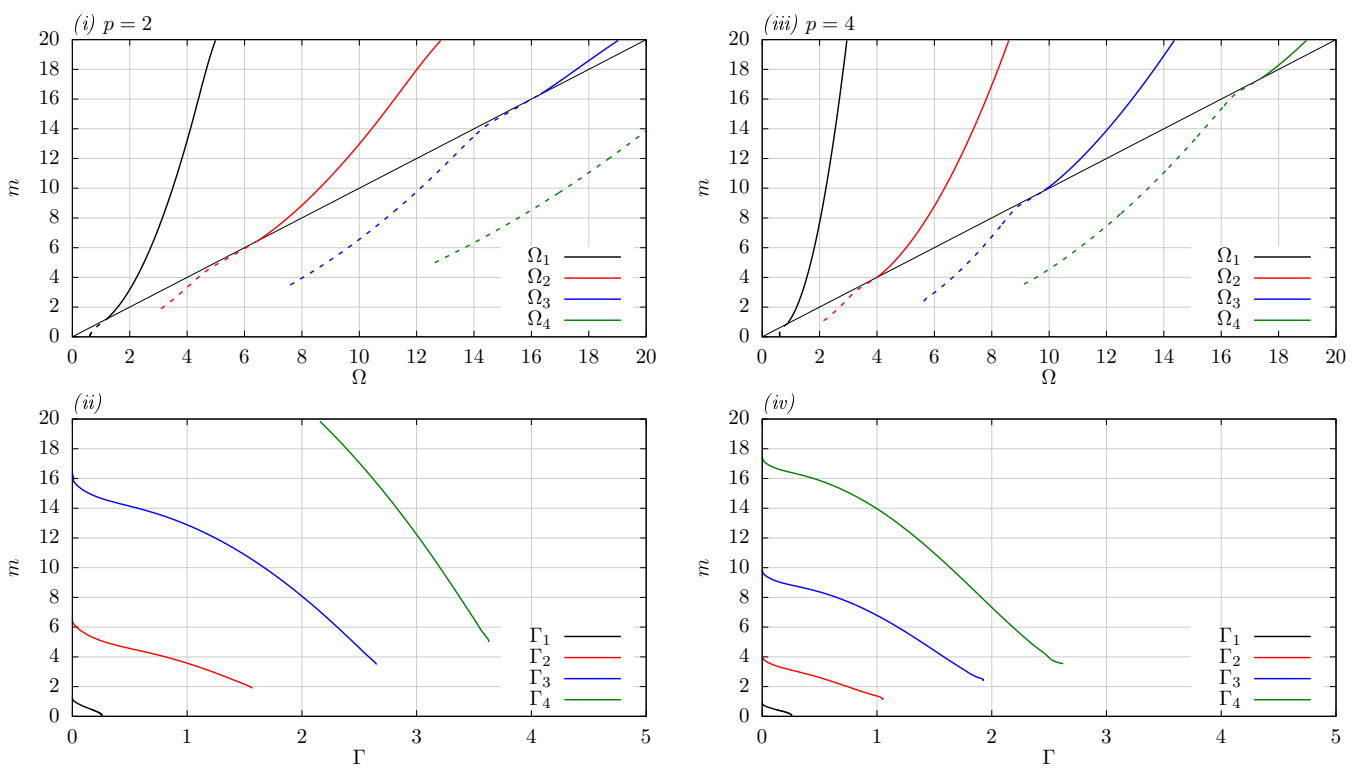

Figure 6. The frequency $\Omega$ and width $\Gamma$ of the lowest resonance mode as a function of the pion mass for the Lin-Piette potentials $p=2$ and $p=4$.
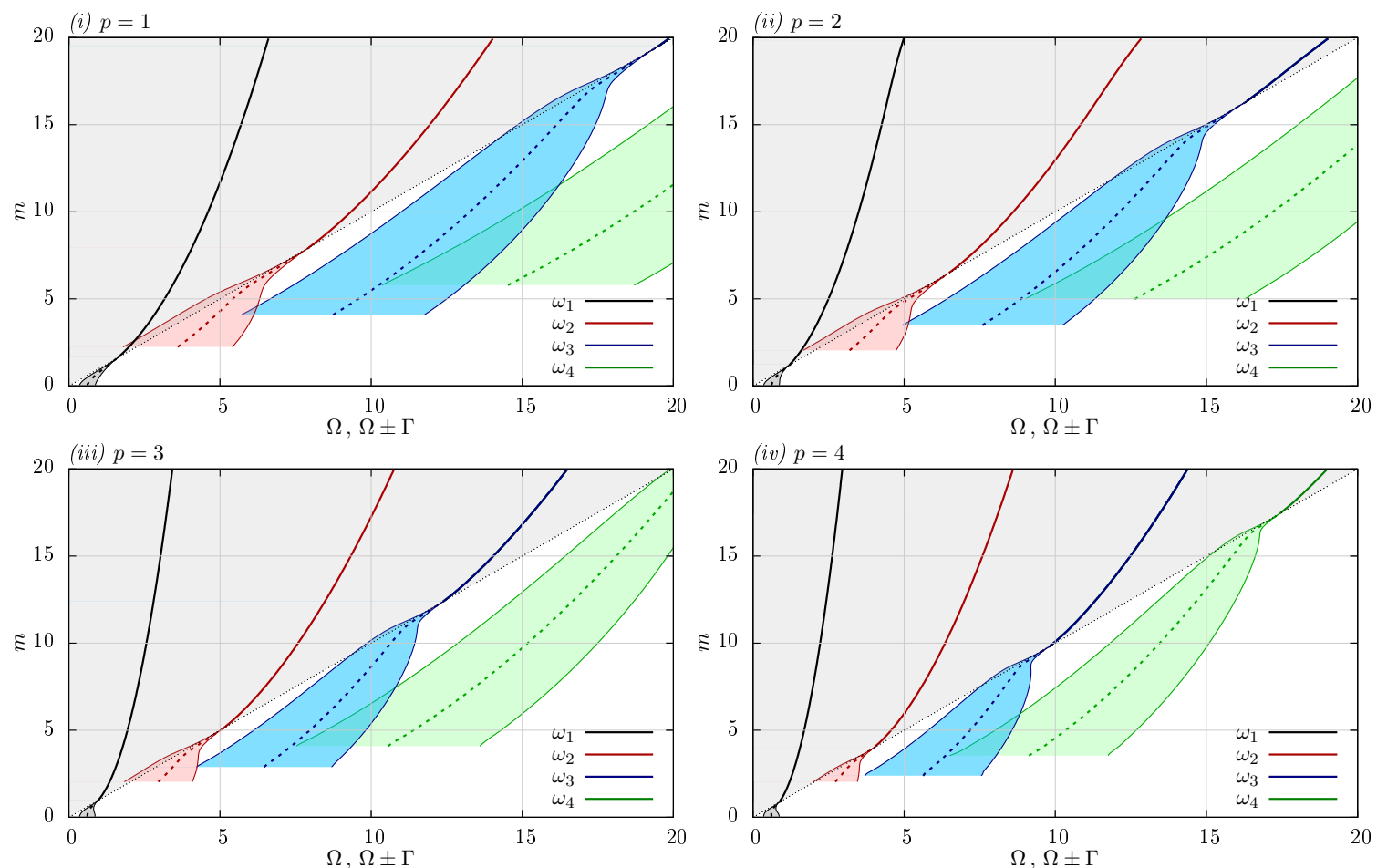

Figure 7. Oscillational and resonance modes for the Lin-Piette potentials $p=1,2,3,4$ with the pion mass $m \in[0,20]$. The real parts of the quasinormal mode frequencies are depicted with dashed lines and their widths (imaginary parts) with shaded regions. 


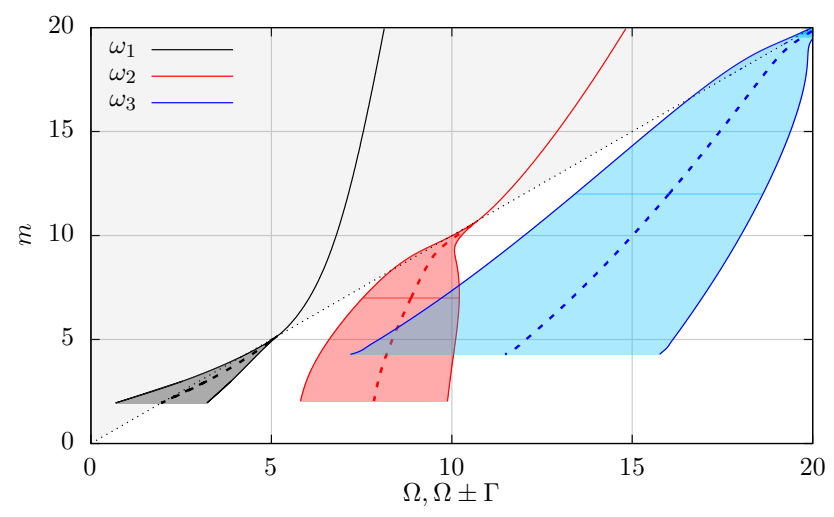

Figure 8. Resonance modes for the unbinding potential with $\alpha=0.95$.

Usually already the second harmonics is above the mass threshold and propagates to spatial infinity, carrying away the energy from the oscillational mode. This results in the decay of the mode (see figure 9 for the $p=2$ Lin-Piette potential for $m=4.5$ ). However, for certain choices of parameters the second harmonics can still be below the mass threshold and only the third one propagates. Since higher harmonics are described by higher order perturbations, in this case the mode decays much slower than in the first case (see figure 10 for the $p=2$ Lin-Piette potential for $m=5.5$ ). This phenomenon was recently observed in $[57,58]$.

\section{The full Skyrme model and the role of the sextic term}

In this section, we consider the full Skyrme model where the sextic term is included. Moreover, we consider the family of potentials $\mathcal{U}^{(p)}$ introduced above. We remark that the resonance structure in the pure BPS Skyrme model and its dependence on a particular form of the potential was carefully investigated in [50] (see also [56]).

From a qualitative point of view, the asymptotics is completely dictated by the potential contribution. In fact, $Q_{\infty}$ remains unchanged. Therefore, the existence of a mass threshold at which possible oscillating modes cease to exist continues to hold, as well.

In figure 11 (right panel) we show the effective potential in the case of the usual Skyrme model and for several values of the $\epsilon$ constant (while other couplings are fixed). Qualitatively, the inclusion of the sextic term to the usual Skyrme model (with the previously chosen values of the parameters) leads to two effects: 1) the bottom of the little well is raised above 0 . This happens at least for sufficiently large $\epsilon ; 2)$ the width of the well is widened. The first effect means that the sextic term prevents the appearance of a negative energy bound state and therefore contributes to the stability of the Skyrmion. The second effect results in lowering the value of the critical mass below which there is no oscillating mode, and in increasing the number of oscillating modes, see figure 12 and tables 2, 3. Furthermore, the resonance modes become narrower. These two effects, which are visible for higher values of $\epsilon$ (see for example figure 13) do not occur for small perturbations of the $\mathcal{L}_{024}$ model. Indeed, for small $\epsilon$ (below 1 ) the critical mass for the first oscillating mode grows, which means that inclusion of a small fraction of the sextic term can lead to 

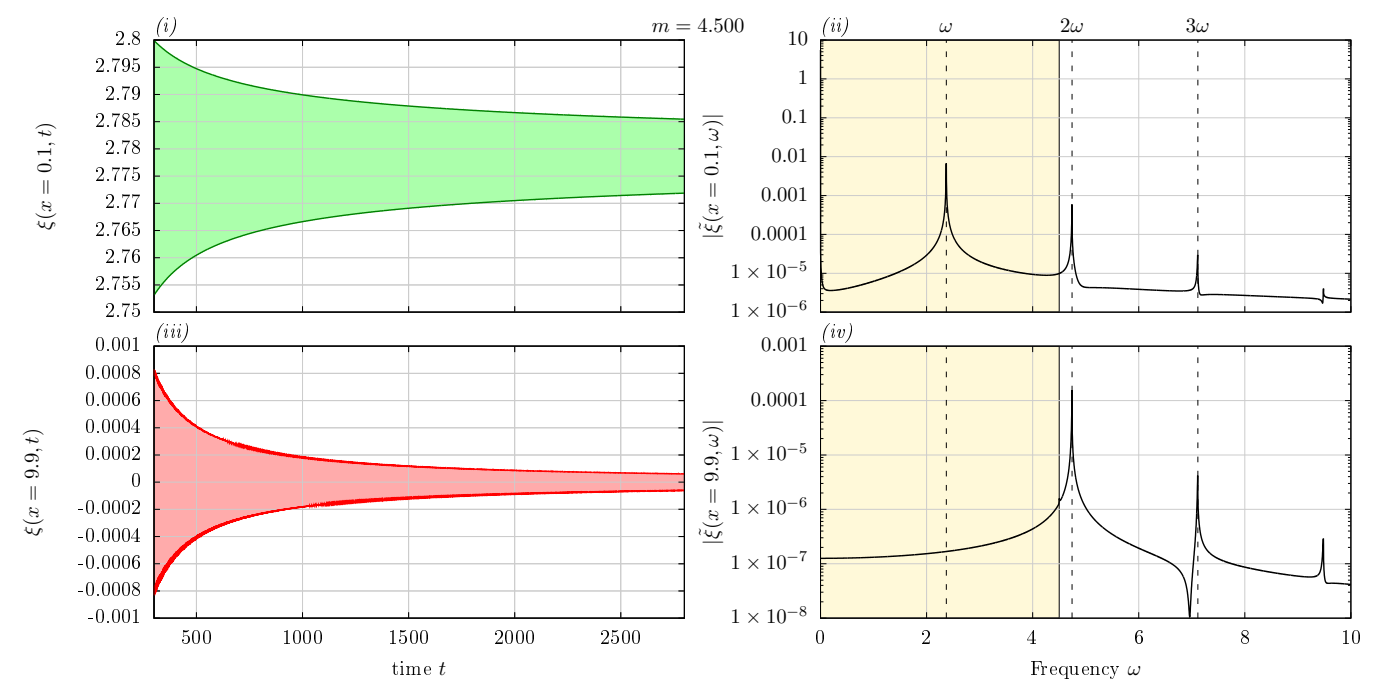

Figure 9. Normal decay of the oscillational mode for the $p=2$ case through the second harmonics. The left panels show the time dependence of the field oscillations near the center (i) and at a certain distance from the skyrmion core (iii). The right panels show the power spectrum of the oscillations.
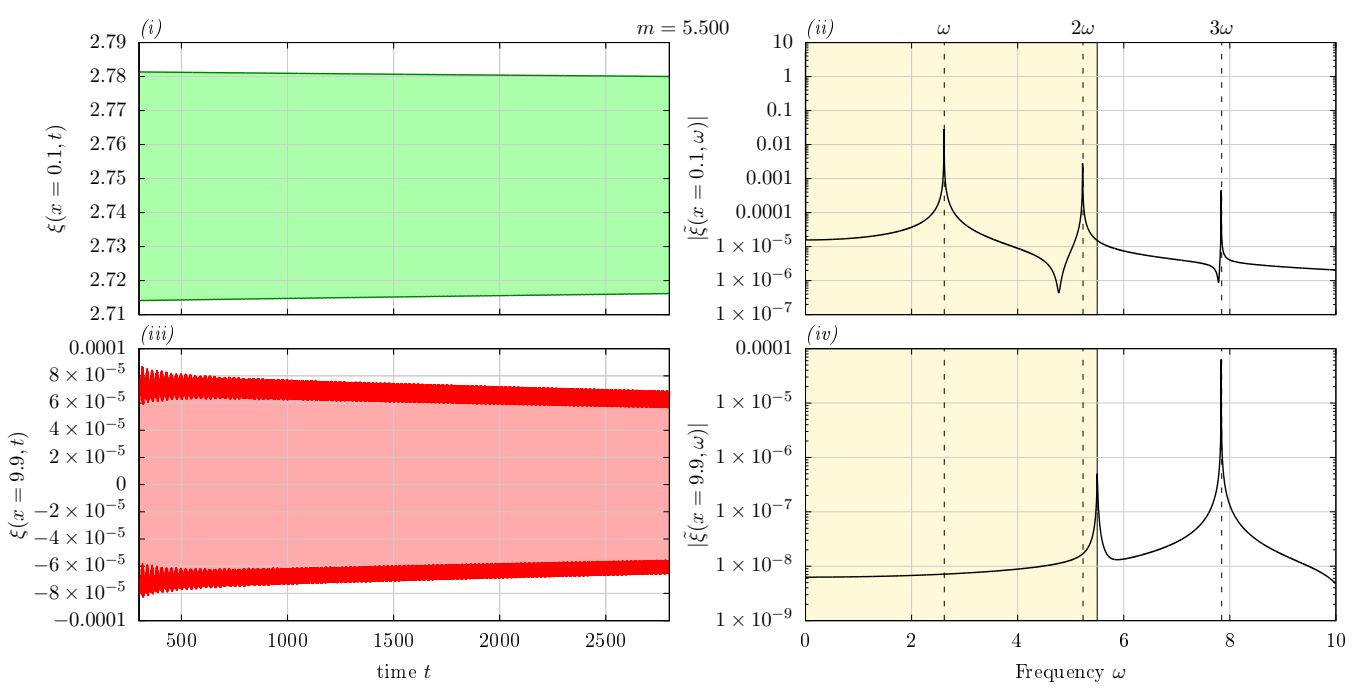

Figure 10. Slow decay of the oscillational mode for the $p=2$ case through the third harmonics. The left panels show the time dependence of the field oscillations near the center (i) and at a certain distance from the skyrmion core (iii). The right panels show the power spectrum of the oscillations. Note that the second harmonics this time is below the mass threshold and cannot propagate (no sign of it in (iv)).

the disappearance of some oscillating modes (and the appearance of resonance modes) see figure 14. The equivalent result for the full numerics is seen in figure 15 . The linear resonance spectra for different pion mass values are shown in figure 16. As we see, the inclusion of the sextic term has a substantial and quite nonlinear impact on the structure of the quasi-normal modes. Furthermore, for the chosen potentials, the differences between the widths of the first few resonances, again, are too big to reproduce the experimental data. 

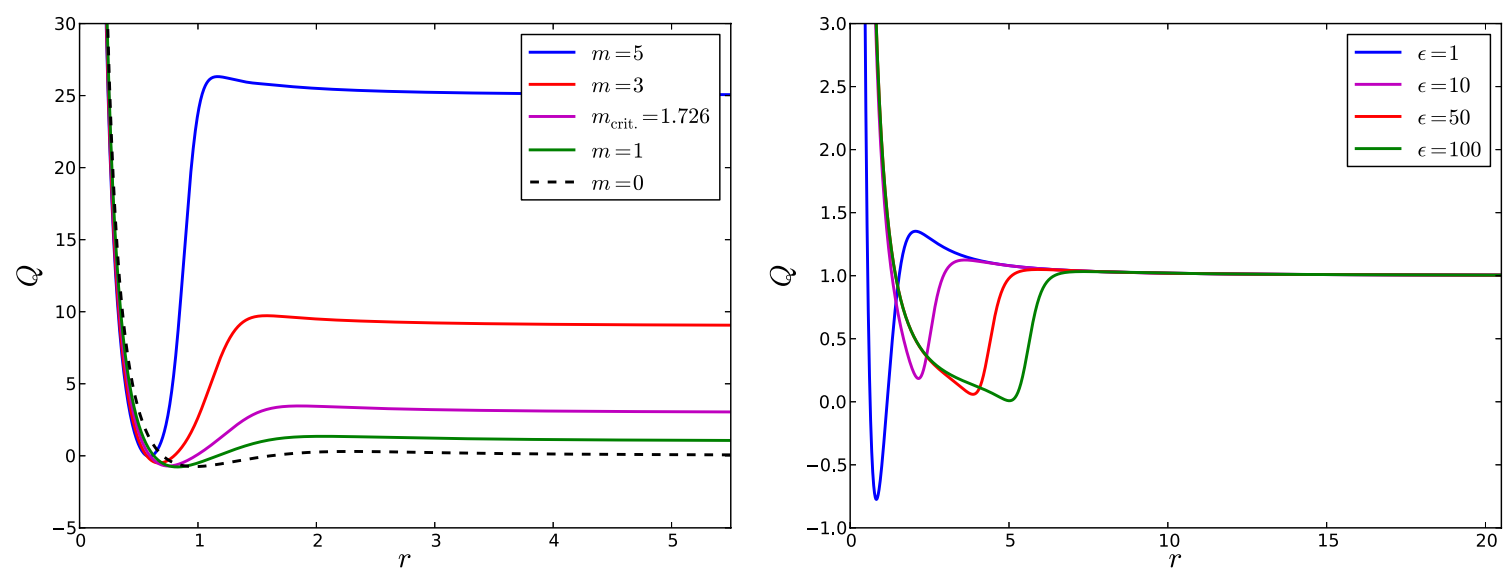

Figure 11. Effective potential $Q$ for the $L_{2460}$ Skyrme model with the usual Skyrme potential. Left: $\epsilon=1$ and different value of the mass $m$. Right: $m=1$ and different values of $\epsilon$.
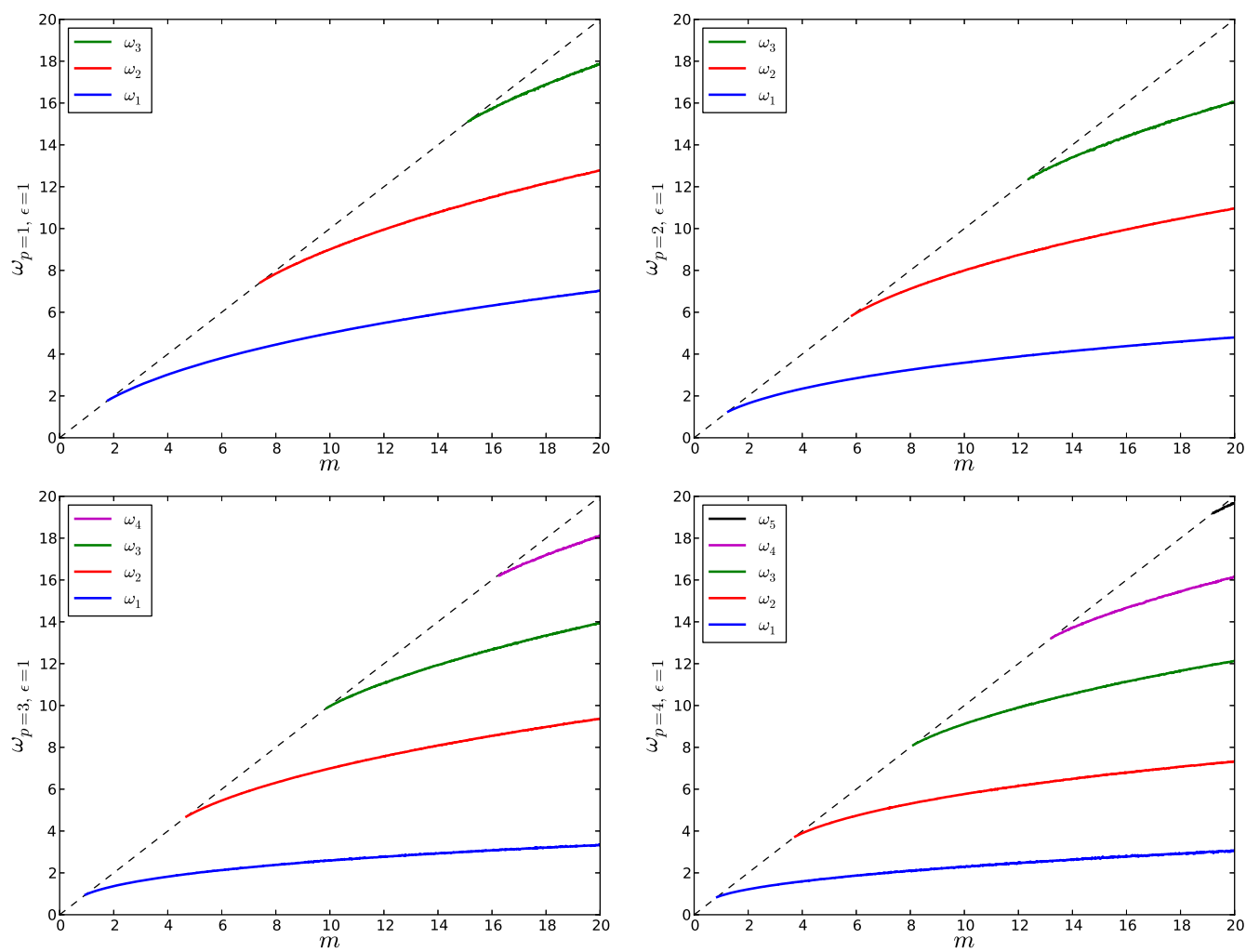

Figure 12. The lowest oscillating modes for the $L_{2460}$ Skyrme model $(\epsilon=1)$ with the Lin-Piette potentials $\mathcal{U}^{(p)}, p=1,2,3,4$, as a function of the pion mass $m$. 

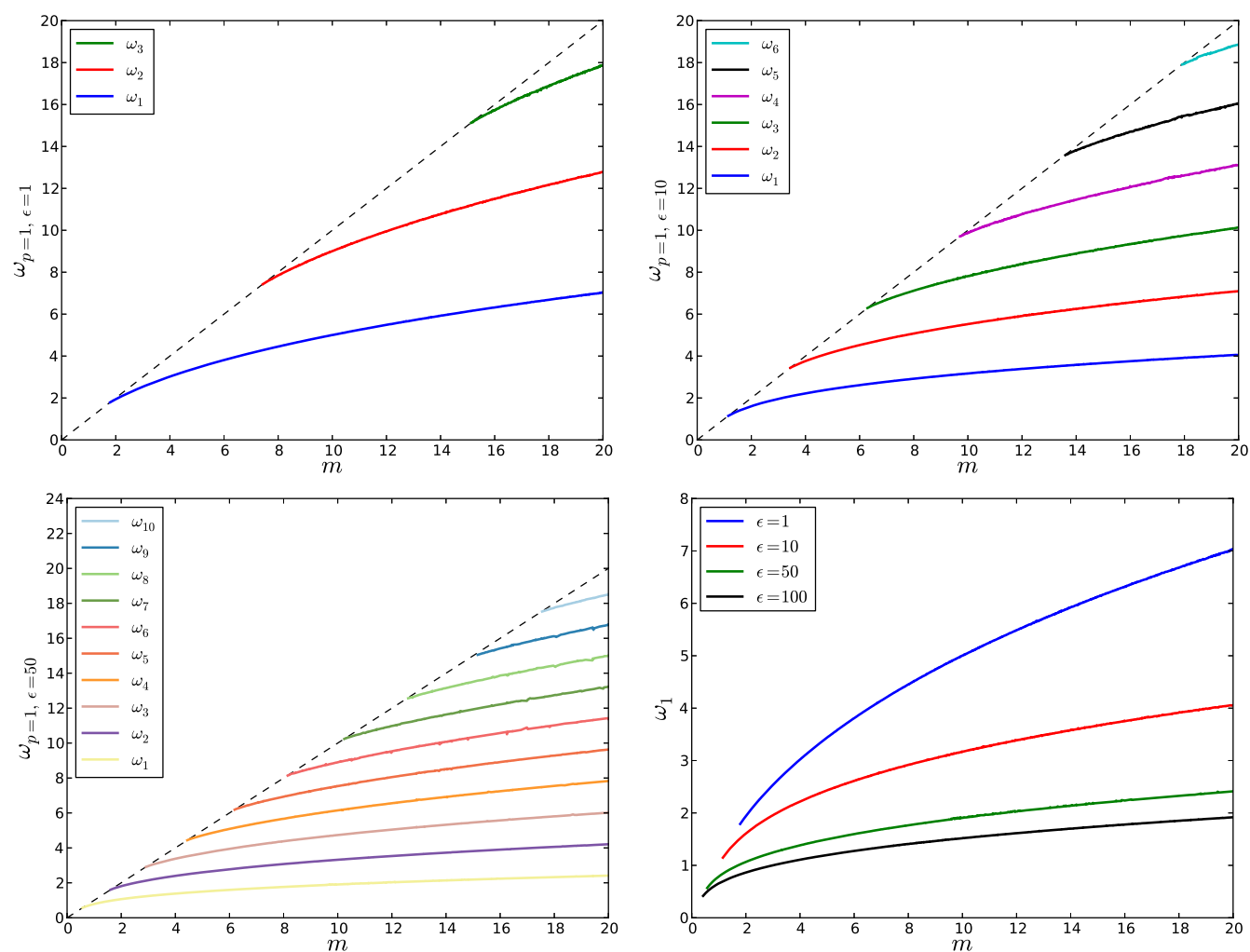

Figure 13. The lowest oscillating modes for the $L_{2460}$ Skyrme model with the usual Skyrme potential $\mathcal{U}^{(p=1)}$ for $\epsilon=1,10,50$, as a function of the pion mass $m$. In the last panel, we show the first oscillating mode $\omega_{1}$ for different $\epsilon$.
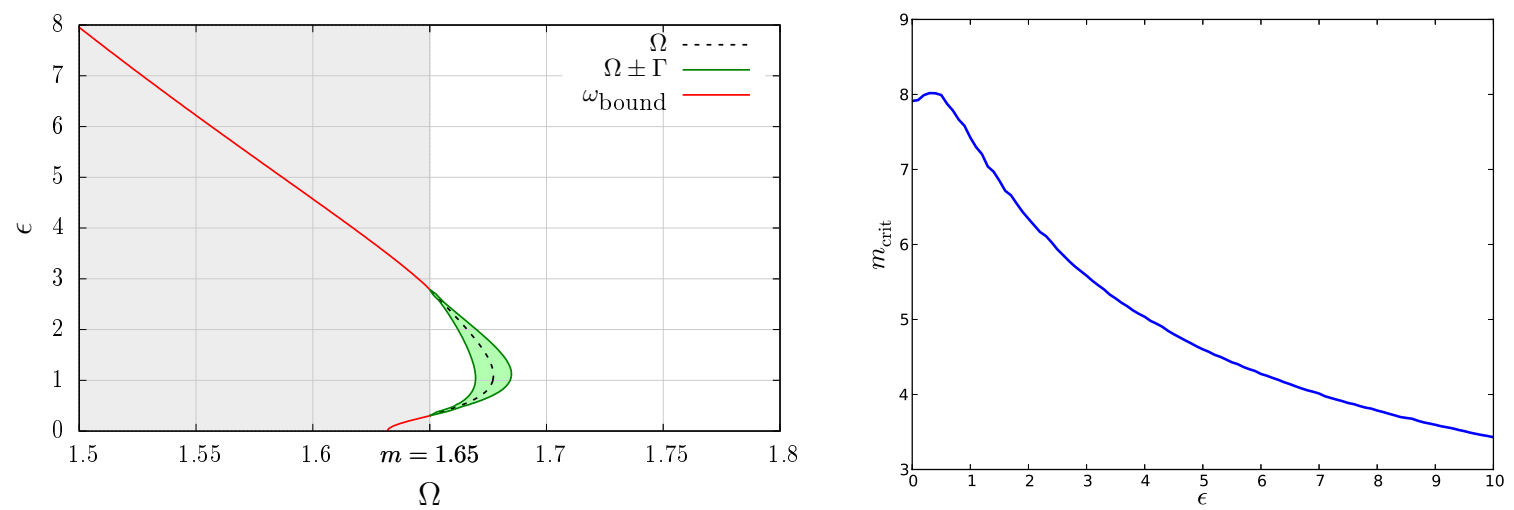

Figure 14. Left: the first oscillating/resonance mode for $m=1.65$. Right: value of the critical mass for the second oscillating mode. 


\begin{tabular}{|c|ccccc|}
\hline & $n=1$ & $n=2$ & $n=3$ & $n=4$ & $n=5$ \\
\hline$\epsilon=0$ & 1.59 & 7.98 & 19.37 & - & - \\
\hline$\epsilon=1$ & 1.79 & 7.42 & 15.12 & - & - \\
\hline$\epsilon=10$ & 1.14 & 3.43 & 6.29 & 9.69 & 13.52 \\
\hline$\epsilon=50$ & 0.56 & 1.60 & 2.90 & 4.44 & 6.18 \\
\hline
\end{tabular}

Table 2. Critical masses for the first five oscillating modes for the full Skyrme model with the usual Skyrme potential $\mathcal{U}^{(p=1)}$. No value denotes that the critical point occurs above $m=20$.

\begin{tabular}{|l|ccccc||c|ccccc|}
\hline & $n=1$ & $n=2$ & $n=3$ & $n=4$ & $n=5$ \\
\hline$p=1$ & 1.59 & 7.99 & 19.38 & - & - & & $n=1$ & $n=2$ & $n=3$ & $n=4$ & $n=5$ \\
$p=2$ & 1.18 & 6.47 & 16.22 & - & - & $p=1$ & 1.79 & 7.42 & 15.12 & - & - \\
$p=2$ & 1.23 & 5.84 & 12.36 & - & - \\
$p=3$ & 0.95 & 5.07 & 12.38 & - & - & $p=3$ & 0.96 & 4.69 & 9.86 & 16.25 & - \\
$p=4$ & 0.85 & 3.98 & 9.77 & 17.56 & - & $p=4$ & 0.83 & 3.74 & 8.10 & 13.23 & 19.19 \\
\hline
\end{tabular}

Table 3. Critical masses for the full Skyrme model with the Lin-Piette potentials. No value denotes that the critical point occurs above $m=20$. Left: $\epsilon=0$. Right: $\epsilon=1$.

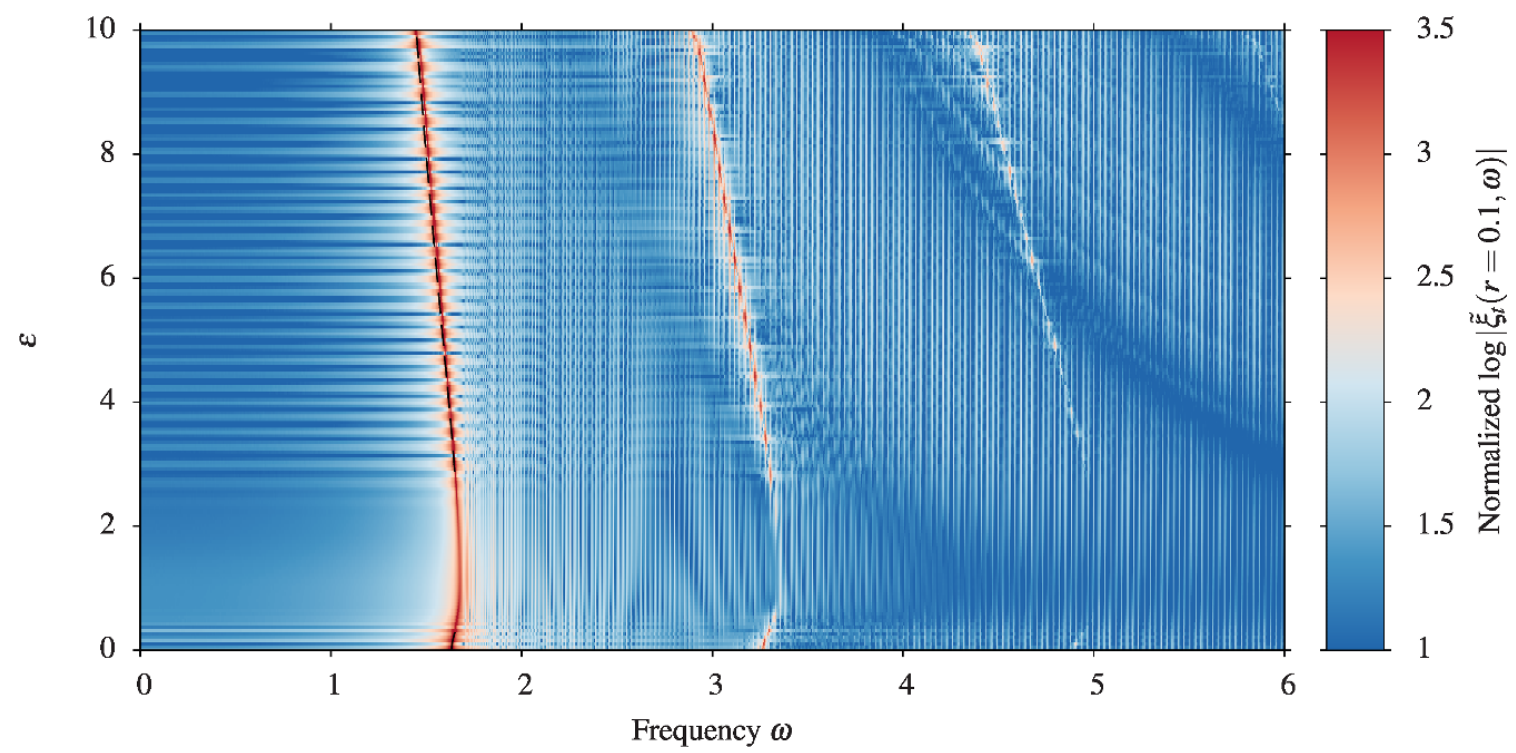

Figure 15. FFT scan of the full dynamics for the full Skyrme model $\mathcal{L}_{0246}$ with the usual potential and $m=1.65$. A window where the first oscillating mode is replaced by a resonance is visible. The dashed line is the oscillating mode. 


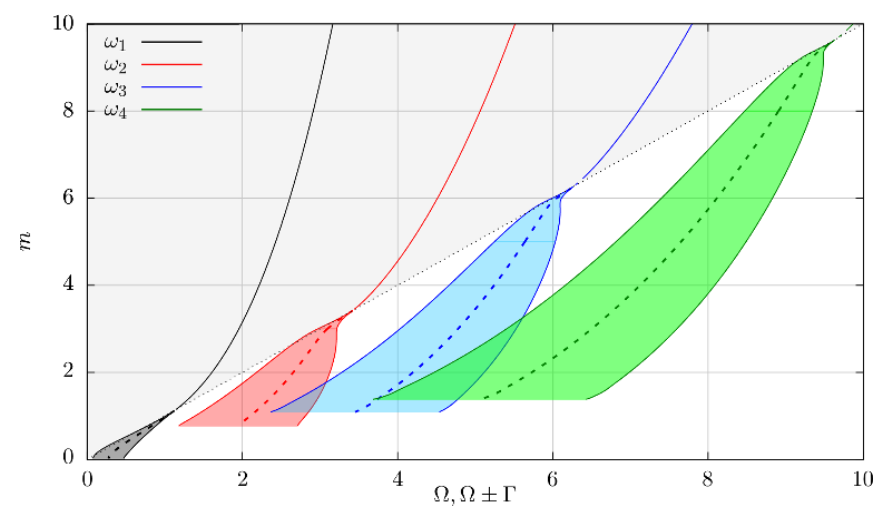

Figure 16. Resonance modes for the full Skyrme model with $\epsilon=10$ and the usual pion mass potential.

\section{Reconstruction}

Up to now we have been considering models with a given potential $\mathcal{U}(\xi)$. We found static Skyrmionic solutions in the charge one sector. Then we perturbed the static solutions and obtained the effective potential $Q(r)$ which is the core ingredient in the linear perturbation theory. Finally, we analysed the spectral structure of the potential $Q(r)$ by solving the appropriate Sturm-Liouville equation, obtaining resonances or bound states. Obviously, qualitative as well as quantitative properties of the spectra are determined by a particular form of the effective potential $Q(r)$.

Since there are many acceptable potentials in the Skyrme framework, provided they lead to the physical pion mass and reasonable binding energies, one can pose the opposite problem. Namely, for a given (qualitatively or even quantitatively reasonable) vibrational spectrum, which results from a given effective potential $Q(r)$, to reconstruct the original potential $\mathcal{U}$. In this section we show how the full nonlinear model can be obtained from the linearised effective potential $Q(r)$.

\section{$5.11+1$ dimensional example}

First, we start with a simple example of one scalar field in $1+1$ dimensions. Then, the equation for the static solution is

$$
-\xi_{0, x x}+\mathcal{U}_{\xi}\left(\xi_{0}\right)=0
$$

The linearised equation describing small perturbations around the static solution can be found as

$$
-\eta_{x x}+Q(x) \eta=\omega^{2} \eta
$$

where

$$
Q(x)=\mathcal{U}_{\xi \xi}\left(\xi_{0}(x)\right) .
$$

For concreteness we assume

$$
Q(x)=m^{2}-\frac{2}{\cosh ^{2} x} .
$$


Introducing a new variable $\mathcal{W}=\mathcal{U}_{\xi}$, we can write the following system of equations for $\mathcal{W}$ and $\xi$, equivalent to eqs. (5.1) and (5.3):

$$
\left\{\begin{array}{l}
\mathcal{W}_{\xi}=Q, \\
\xi_{x x}=\mathcal{W} .
\end{array}\right.
$$

After changing the variable $\mathcal{W}_{\xi}=\mathcal{W}_{x} / \xi_{x}$ the system can be written as

$$
\left\{\begin{array}{l}
\mathcal{W}_{x}=Q \xi_{x}, \\
\xi_{x x}=\mathcal{W} .
\end{array}\right.
$$

Formally, the system can be solved after applying appropriate boundary conditions. Namely, $\xi$ has to satisfy the usual topological boundary conditions. For symmetric potentials we can assume that $\xi(0)=\pi$, which is the maximum of the potential (topological zero) and $\xi(\infty)=\xi_{v a c}=0$ which is the real vacuum (minimum of the field theory potential). Since both of these points are extrema of the potential, $\mathcal{U}_{\xi}=\mathcal{W}=0$ at these points. The problem to solve is a two point boundary condition problem. Starting from $x=0$, we have to satisfy two conditions at $x=\infty$. To do that, two (shooting) parameters have to be used. One of them is $\xi_{x}(0)$. The second is the choice of $m$.

In this trivial example, the second equation can be differentiated and we obtain

$$
\xi_{x x x}=\mathcal{W}_{x}=Q \xi_{x}
$$

This is the linearised equation for $\eta=\xi_{x}$ with $\omega=0$. Actually, $\xi_{x}$ has an interpretation as the translational mode of the soliton $\xi(x+a) \approx \xi(x)+a \xi_{x}(x)+\mathcal{O}\left(a^{2}\right) . \xi_{x}$ is a small perturbation, and since the model has the translation symmetry, $\xi+a \xi_{x}$ is also a static solution, so $\omega$ must be zero. In our working example, the potential has a single bound mode $\eta(x)=1 / \cosh (x)$ with the frequency $\omega^{2}=m^{2}-1$. So $m=1$. Now we have to solve the equation $\xi_{x}=c_{1} / \cosh (x)$, which fortunately has a closed form

$$
\xi(x)=2 c_{1} \tan ^{-1}(\exp (x))+c_{2} .
$$

Applying boundary conditions we obtain $c_{1}=-2, c_{2}=2 \pi$. Now we know the profile of the static soliton solution. From the equation $\mathcal{W}=\xi_{x x}=\mathcal{U}_{\xi}$ we can obtain:

$$
2 \xi_{x} \xi_{x x}=2 \xi_{x} \mathcal{U}_{\xi} \quad \Rightarrow \quad \mathcal{U}_{x}=\frac{1}{2}\left(\xi_{x}^{2}\right)_{x} \quad \Rightarrow \quad \mathcal{U}(x)=\frac{1}{2} \xi_{x}^{2}+C .
$$

The last equation can be treated as an implicit (parametric) equation for the potential $\mathcal{U}(\xi)$. The final form can be untangled by using the relation that $\frac{1}{2} \xi_{x}^{2}=1+\cos \xi$ and setting $C=0$. The obtained example is in fact the shifted sine-Gordon model

$$
\mathcal{U}(\xi)=1-\cos \xi
$$

Similarly, from the effective potential

$$
Q=m^{2}-\frac{6}{\cosh ^{2}(x)}
$$

the well known $\phi^{4}$ model can be obtained. 
The working example was a simple $1+1$ dimensional model which can be easily separated. However, we have also tested the procedure by solving numerically the system (5.6) with the boundary conditions $\mathcal{W}(0)=\mathcal{W}(\infty)=\xi(\infty)=0$ and $\xi(0)=\pi$.

\subsection{Skyrme model from the effective potential}

In principle, we could repeat the procedure described in the previous subsection for Skyrmions and obtain $\mathcal{U}$ from an effective potential $Q$ with the desired spectral properties. However, the procedure requires the solution for the translational mode. Our original theory is translation invariant, but our hedgehog ansatz is not. Moreover, we don't expect that at any point we could find a closed form of the potential, therefore, we focus on the numerical procedure rather than on the analytical approach.

The equations to be solved are the following (analogously to eqs. (5.6) in the $1+1$ dimensional case)

1. The equation for the static solution $\xi_{0}$ of eq. (2.8)

2. $Q=Q_{0}(r, \xi)+Q_{\mathcal{U}}\left(r, \xi, \mathcal{W}, \mathcal{W}_{\xi}\right)$ where $\mathcal{W}=\mathcal{U}_{\xi}$.

Now, the strategy is as follows. First, we try to find an effective potential $Q$ which leads to narrow resonance modes with comparable widths. For example, we found the following structure of resonances

$$
\begin{aligned}
& \Omega_{1}+i \Gamma_{1}=1.710+0.250 i \\
& \Omega_{2}+i \Gamma_{2}=1.880+0.250 i \\
& \Omega_{3}+i \Gamma_{3}=2.384+0.228 i \\
& \Omega_{4}+i \Gamma_{4}=2.869+0.336 i
\end{aligned}
$$

for the piecewise flat effective potential shown in panel (ii) of figure 17 (with the usual dimensionally induced $\frac{2}{r^{2}}$ core included; the reason for choosing piecewise flat potentials $Q$ is explained in the next subsection). In a second step, we perform the (numerical) reconstruction of the generalised Skyrme model and, in particular, of the field theoretic potential $\mathcal{U}$, as explained above. In figure 17 we present the result of this (numerical) reconstruction procedure. For small $\epsilon$, the resulting field theoretical potential is very peaked at the anti-vacuum, which corresponds to a significant (probably unphysical) concentration of the energy density at the origin. This unpleasant fact is cured if we increase the value of the coupling constant $\epsilon$ multiplying the sextic term, i.e., once we move the model towards the near-BPS Skyrme model $[59,60]$. Then, it turns out that the energy density becomes flatter.

\subsection{Roper reconstruction details}

Here, we use the reconstruction procedure described in the previous section for parametrized families of effective potentials $Q$, and fit these parameters to physical observables of the nucleon, like the Roper resonances, the nucleon mass and the isoscalar electric charge radius of the nucleon. For the reconstruction we have decided to use piecewise flat effective 

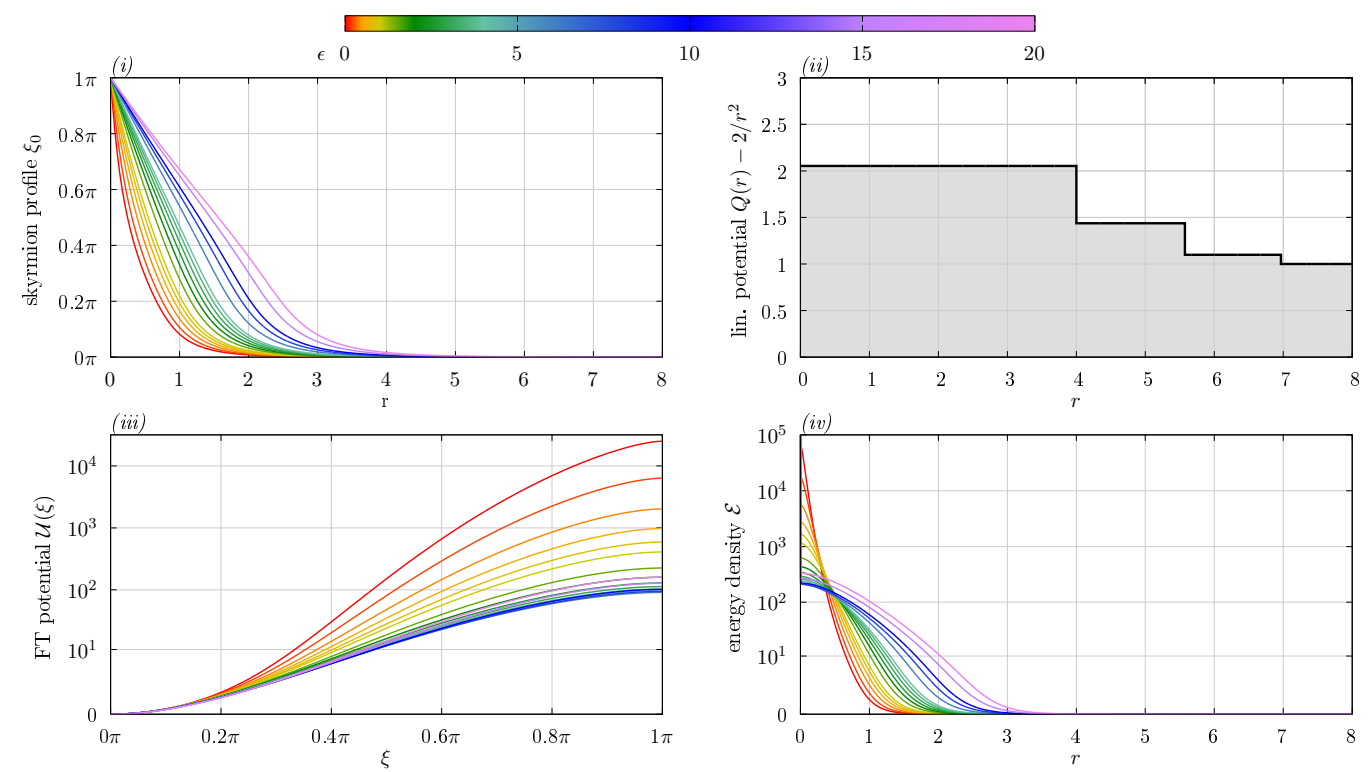

Figure 17. Reconstruction of the field theoretic potential $\mathcal{U}$ for the full Skyrme model $\mathcal{L}_{0246}$. (i) profiles of the solutions; (ii) the effective potential $Q$ with the 3-dimensional universal repulsive core subtracted; (iii) the field theoretical potentials $\mathcal{U}$; (iv) the energy densities. The structure of the resonances is described in the text.

potentials (plus the usual $2 / r^{2}$ term) because the resulting problem can be solved partly analytically. The potential can then be written in the form

$$
Q\left(r ;\left\{Q_{n}, L_{n}\right\}\right)=\frac{2}{r^{2}}+\sum_{n=0}^{N-1} Q_{n} H\left(r-r_{n}\right) H\left(r_{n+1}-r\right)
$$

where $\left\{Q_{n}, L_{n}\right\}, n=0, \ldots N-1$, are the parameters of the potential, the height and length of each segment,

$$
r_{n}=\sum_{i<n} L_{i}
$$

and $H(x)$ is the Heaviside step function. Further, $r_{0}=0<r_{1}<r_{2}<\ldots<r_{N}=\infty$, the potential consists of $N$ steps, and there are $N-1$ matching points $r_{1}<r_{2}<\ldots r_{N-1}$.

The solutions are known

$$
u_{n}(r)=A_{n} u_{1, n}(r)+B_{n} u_{2, n}(r), \quad \text { where } \quad r_{n} \leq r \leq r_{n+1}
$$

and

$$
u_{1, n}(r)=\left(1+\frac{i}{k_{n} r}\right) e^{i k_{n} r} \quad \text { and } \quad u_{2, n}(r)=\left(1-\frac{i}{k_{n} r}\right) e^{-i k_{n} r}, \quad k_{n}=\sqrt{\omega^{2}-Q_{n}} .
$$

The solutions should have the same values and derivatives at the matching points. The matching can be performed with the help of the transition matrix defined as $(n=1, \ldots, N-1)$

$$
M\left(k_{n}, k_{n-1}, r_{n}\right)=\left[\begin{array}{ll}
u_{1, n} & u_{2, n} \\
u_{1, n}^{\prime} & u_{2, n}^{\prime}
\end{array}\right]_{r_{n}}^{-1}\left[\begin{array}{ll}
u_{1, n-1} & u_{2, n-1} \\
u_{1, n-1}^{\prime} & u_{2, n-1}^{\prime}
\end{array}\right]_{r_{n}}
$$


which transfers the solution from one segment to the next,

$$
\left[\begin{array}{l}
A_{n} \\
B_{n}
\end{array}\right]=M\left(k_{n}, k_{n-1}, r_{n}\right)\left[\begin{array}{l}
A_{n-1} \\
B_{n-1}
\end{array}\right] .
$$

The asymptotic solution can be found by simply multiplying these matrices in the appropriate order (larger $n$ on the left) and acting on the initial conditions

$$
\left[\begin{array}{l}
A_{N} \\
B_{N}
\end{array}\right]=\prod_{n=N}^{1} M\left(k_{n}, k_{n-1}, r_{n}\right)\left[\begin{array}{l}
A_{0} \\
B_{0}
\end{array}\right] .
$$

The resonance condition $A_{N} / B_{N}=0$ for $A_{0}=B_{0}=1$ is just an algebraic equation for $\omega$. Solving such an equation numerically is much simpler than finding the resonance modes integrating the appropriate ODEs. Using the procedure described in the previous section, we can reconstruct the full field theory potential $\mathcal{U}(\xi)$ from the linearized potential $Q(r)$.

For the fitting procedure, we first set the pion mass and pion decay constant to their physical values, $m_{\pi}=138 \mathrm{MeV}$ and $f_{\pi}=186 \mathrm{MeV}$. The model has two more independent parameters $e$ and $\epsilon$ which we use as independent variables. $e$ sets the energy and length scales

$$
\ell=\frac{2 \hbar}{f_{\pi} e}
$$

Our procedure of finding the appropriate model is the following. We choose the parameters describing the model $(e, \epsilon)$ and the effective potential $\left\{Q_{n}, L_{n}\right\}$ as independent variables which we vary to obtain the most physical model.

1. The value of $e$ sets all scales needed for the asymptotic value of the potential

$$
Q(r \rightarrow \infty) \rightarrow m^{2}=\frac{2 m_{\pi}}{f_{\pi} e} .
$$

2. From a set of potential steps $\left\{Q_{n}, L_{n}\right\}$ we calculate the resonances and scale them to their physical values

$$
\omega_{i, \mathrm{ph}}=\frac{f_{\pi} e}{2} \omega_{i}
$$

Here we chose $N=3$ (two steps) in all examples except for example 4 (where $N=4$, leading to much slower convergence).

3. From $Q(r)$ we reconstruct the potential $\mathcal{U}$, find the isoscalar charge radius $R_{c}$ and the energy (mass) of the nucleon $M$.

4. We calculate a function which measures the weighted relative distances (deviations) from the desired values:

$$
\begin{aligned}
G\left(e, \epsilon,\left\{Q_{n}, L_{n}\right\}\right)= & w_{M}^{2} \frac{\left(M-M_{\mathrm{ph}}\right)^{2}}{M_{\mathrm{ph}}^{2}}+w_{R}^{2} \frac{\left(R_{c}-R_{c, \mathrm{ph}}\right)^{2}}{R_{c, \mathrm{ph}}^{2}} \\
& +\sum_{n=1}^{3}\left(w_{n, r}^{2} \frac{\left(\Omega_{n}-\Omega_{n, \mathrm{ph}}\right)^{2}}{\Omega_{n, \mathrm{ph}}^{2}}+w_{n, i}^{2} \frac{\left(\Gamma_{n}-\Gamma_{n, \mathrm{ph}}\right)^{2}}{\Gamma_{n, \mathrm{ph}}^{2}}\right) .
\end{aligned}
$$


For the desired (physical) values we choose $M_{\mathrm{ph}}=932 \mathrm{MeV}$ (one-fourth of the mass of the helium nucleus, because the helium does not receive spin/isospin contributions and, therefore, is suitable for a comparison with the classical Skyrmion mass), $R_{c, \mathrm{ph}}=0.72 \mathrm{fm}$, and the Roper values $\Omega_{1}=508 \mathrm{MeV}, \Omega_{2}=778 \mathrm{MeV}, \Omega_{3}=958 \mathrm{MeV}$, as well as $\Gamma_{1}=\Gamma_{2}=\Gamma_{3}=300 \mathrm{MeV}$ (taking into account the rather large errors for $\Gamma_{2}$ and $\Gamma_{3}$ ).

Further, the $w_{k}$ are the relative weights of each observable.

5. We vary the variables $\left(e, \epsilon,\left\{Q_{n}, L_{n}\right\}\right)$ and repeat the steps $2-4$ to minimize the above distance function.

In principle, by choosing at least the same number of parameters $\left(e, \epsilon,\left\{Q_{n}, L_{n}\right\}\right)$ as the known values $\left(M, R_{c}, \omega_{i}\right)$ to which we want to match our model, we should be able to find a minimum giving $G=0$. In practice, we have found this a very difficult task. The function $G$ can have discontinuities and long and twisted valleys. Sometimes the whole structure of resonances can change dramatically when the height of the potential is changed even insignificantly. Moreover, some values are more important (measured with higher precision in experiments) so it might be better sometimes to change the weights. For example, the widths of the resonances are known with a considerable error, therefore the weights could be lowered. Different choices of weights gave us different minimized values of $G$.

In table 4 we show some of the values for the observables we found. In the different examples, both the initial values of the parameters $\left(e, \epsilon,\left\{Q_{n}, L_{n}\right\}\right)$ and the weights $w_{k}$ where chosen slightly differently.

- In the first example all weights are equal, $w_{i}=1$. As a result, the first resonance $\Omega_{1}$ has the largest discrepancy from the physical value. However, this is the resonance which is measured with the highest accuracy so our model should reconstruct it also with the highest accuracy. Also, the mass of the nucleon has a rather large error.

- For the second and the third example, we increased the weights $w_{M}=w_{1, r}=10$ to cure the largest errors of the most accurate physical observables. And, indeed, the errors are significantly reduced at the cost of the accuracy of especially $\Omega_{3}$ and $\Gamma_{3}$. The difference between the effective potentials in examples 2 and 3 is due to different (random) starting points for the minimization procedure.

- In the fourth example, we increased the number of pieces (segments) of the potential $Q$ but again with equal weights. The increase of the number of degrees of freedom did not help much but caused the minimization procedure to converge even slower.

- The last example was done for increased weights for the mass and real parts of the resonance frequencies $w_{M}=w_{n, r}=10$.

Figure 18 shows the reconstructed skyrmion profiles $\xi(r)$, potential $Q(r)$, field theory potential $\mathcal{U}(\xi)$ and the energy density inside the skyrmion. Note that the profiles are almost indistinguishable. However, the zoomed plot in figure 18, panel (ii) shows that there are 


\begin{tabular}{|c|c|c|c|c|c|c|c|c|c|c|}
\hline & \multicolumn{2}{|c|}{ Example 1} & \multicolumn{2}{|c|}{ Example 2} & \multicolumn{2}{|c|}{ Example 3} & \multicolumn{2}{|c|}{ Example 4} & \multicolumn{2}{|c|}{ Example 5} \\
\hline$M$ & 918.65 & $(-1.43 \%)$ & 931.82 & $(-0.02 \%)$ & 930.47 & $(-0.16 \%)$ & 873.74 & $(-6.25 \%)$ & 925.00 & $(-0.75 \%)$ \\
\hline$R_{c}$ & 0.7239 & $(0.54 \%)$ & 0.7199 & $(-0.02 \%)$ & 0.7200 & $(-0.00 \%)$ & 0.7200 & $(-0.00 \%)$ & 0.7200 & $(-0.00 \%)$ \\
\hline$\Omega_{1}$ & 467.89 & $(-7.90 \%)$ & 505.00 & $(-0.59 \%)$ & 506.83 & $(-0.23 \%)$ & 465.37 & $(-8.39 \%)$ & 484.23 & $(-4.68 \%)$ \\
\hline$\Gamma_{1}$ & 301.25 & $(0.42 \%)$ & 292.06 & $(-2.65 \%)$ & 287.90 & $(-4.03 \%)$ & 281.52 & $(-6.16 \%)$ & 297.26 & $(-0.91 \%)$ \\
\hline$\Omega_{2}$ & 758.72 & $(-2.48 \%)$ & 832.37 & $(6.99 \%)$ & 827.26 & $(6.33 \%)$ & 757.35 & $(-2.65 \%)$ & 792.35 & $(1.84 \%)$ \\
\hline$\Gamma_{2}$ & 286.54 & $(-4.49 \%)$ & 293.98 & $(-2.01 \%)$ & 289.32 & $(-3.56 \%)$ & 282.26 & $(-5.91 \%)$ & 296.57 & $(-1.14 \%)$ \\
\hline$\Omega_{3}$ & 1008.39 & $(6.37 \%)$ & 1133.36 & $(19.55 \%)$ & 1123.78 & $(18.54 \%)$ & 1014.10 & $(6.97 \%)$ & 1070.27 & $(12.90 \%)$ \\
\hline$\Gamma_{3}$ & 313.12 & $(4.37 \%)$ & 328.08 & $(9.36 \%)$ & 325.01 & $(8.34 \%)$ & 310.74 & $(3.58 \%)$ & 327.21 & $(9.07 \%)$ \\
\hline$e$ & \multicolumn{2}{|c|}{1.2872} & \multicolumn{2}{|c|}{1.2936} & \multicolumn{2}{|c|}{1.2923} & \multicolumn{2}{|c|}{1.3320} & \multicolumn{2}{|c|}{1.2950} \\
\hline$\epsilon$ & \multicolumn{2}{|c|}{0.01251} & \multicolumn{2}{|c|}{0.09602} & \multicolumn{2}{|c|}{0.08431} & \multicolumn{2}{|c|}{0.10886} & \multicolumn{2}{|c|}{0.07609} \\
\hline
\end{tabular}

Table 4. Values of observables of examples found from minimizing the function $G$, along with the relative deviations from the target values, together with the corresponding fit values of the parameters $e$ and $\epsilon$.
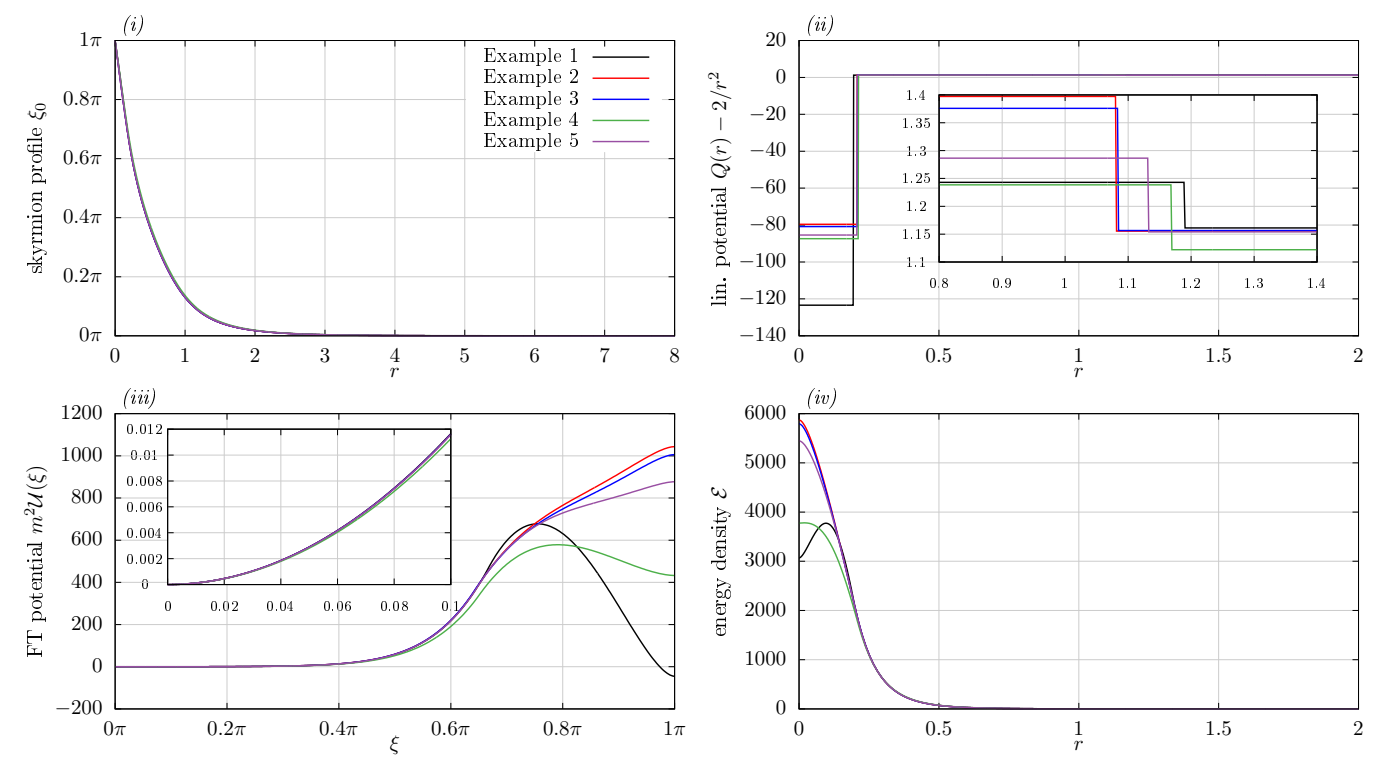

Figure 18. Example solutions found from the reconstruction procedure (i) skyrmion profiles, (ii) the piecewise flat linearized potentials $Q(r)-2 / r^{2}$, zoomed part shows the differences between potentials, (iii) field theoretic potential $\mathcal{U}(\xi)$, zoomed plot shows that indeed $m^{2} \mathcal{U}(\xi) \approx m^{2} \xi^{2}$ for small values of $\xi$, (iv) energy density. The plots are in Skyrme units.

some differences between the effective potentials found in the different examples. Another peculiarity of the examples is the large value of the field theory potential in the center of skyrmions. The rise is so high that the quadratic dependence, $m^{2} \mathcal{U}(\xi) \approx m^{2} \xi^{2}$, valid for small values of $\xi$ looks almost flat (this small region is enhanced by another zoom in panel (iii). 


\section{Summary}

In this paper, we investigated in detail the possibility to describe the Roper resonances within the context of the Skyrme model. We found that the quantitative results strongly depend on the particular Skyrme model under consideration and, specifically, on the potential term. For the standard pion mass potential, it turns out that the Roper resonances are not reproduced very well. In particular, the widths of the higher resonances always come out much larger than for the lowest resonance, in contrast to established experimental facts. From a theoretical point of view there is, however, no reason to restrict to the pion mass potential. The potential should always contain a mass term giving rise to the correct pion mass, but additional terms are perfectly legitimate. Once this possibility is considered, we found that already for rather simple potentials (e.g., the repulsive potential of refs. $[26,27])$ the tension between calculated and experimentally measured Roper resonances can be slightly reduced. However, none of the analysed potentials (with or without the sextic term) provided the correct Roper resonances.

In a second line of investigation, we used an approach which is in some sense inverse to the one described above. Instead of departing from a given Skyrme model, we use a linear, quantum mechanical potential (called "effective potential" in this paper) as a starting point. The determination of the Roper resonances from this linear potential is a much simpler problem. We then developed a reconstruction procedure which allows to reconstruct the Skyrme model (the field theoretical potential $\mathcal{U}$ ) from the initial linear effective potential $Q$. This second approach allows to describe the Roper resonances (and, in a second step, after the Skyrme model is reconstructed, also further observables) with a much higher precision, see table 4 . There are several directions in which this reconstruction method can and should be generalised. First of all, in this paper we only considered piecewise flat linear potentials $Q$, because this simplifies the calculation. Considering more general linear potentials should be interesting. Secondly, we were able to reconstruct the field theoretical potentials $\mathcal{U}$ only numerically. An analytical reconstruction, e.g., by expanding $\mathcal{U}$ in a certain set of basis functions, could be useful, because it would give us more analytical control over the resulting reconstructed Skyrme models. We remark that the reconstruction method for the pure BPS Skyrme model (which had not been used in [50] where only resonances for given potentials were calculated) might be useful to reduce the degeneracy in the potential parameter space and, thus, reconstruct physically viable potentials (although the BPS submodel by itself is insufficient as a low-energy effective field theory for strong interaction physics).

It is one of the main goals of our investigation and related work to determine a generalised Skyrme model (or a restricted class thereof) which provides an effective field theory for nuclear physics allowing to describe both hadronic and nuclear observables precisely. While the results presented here certainly represent further steps in this direction, we think that the observables considered in this paper are not yet sufficient to pin down the correct physical Skyrme model (the correct Skyrme potential and the values of the coupling constants of the derivative terms), and further observables of hadron and nuclear physics are required for this task. First of all, the properties (frequencies and widths) of the resonances are very sensitive to small changes of the effective potential. The resonances, therefore, 
are more useful to precisely determine parameter values whose approximate values have been found already from other sources. Secondly, as can be seen in figure 18 (lower left panel), rather different Skyrme potentials can give rise to rather similar values for the physical observables (resonances, $M, R_{c}$ ). Additional observables of hadron and nuclear physics are, therefore, needed both to resolve this (almost) degeneracy and for a more robust determination of parameter values.

An interesting aspect of this problem is related to the relative strength of the sextic term (the value of the parameter $\epsilon$ ). Indeed, we argued in section 5.2 that higher values of $\epsilon$ should be expected, because they avoid both very peaked energy densities and potentials which rise to very high values for $\xi \rightarrow \pi$. On the other hand, in the examples of fits to physical values of section 5.3 we always found rather small values of $\epsilon$ (see table 4). The resolution of this apparent contradiction is easily understood. In the fit of section 5.3 we did not include observables which would impede peaked energy densities, and the energy densities resulting from the fit are very peaked, see figure 18. The simplest observable avoiding this energy concentration is the energy RMS radius $R_{e}$ defined by (here $E=\int d^{3} x \mathcal{E}$ is the energy)

$$
R_{e}^{2}=E^{-1} \int d^{3} x r^{2} \mathcal{E}
$$

because very peaked energy densities would lead to unacceptably small values for $R_{e}$. We refrained from including this observable into the fit for two reasons. The first reason is that the inclusion of additional observables further complicates the numerical fit procedure. After all, the main purpose of the present article is a proof of principle, and not (yet) a detailed determination of the correct physical Skyrme model of nuclear and hadron physics. The second reason is that there does not seem to exist a direct measurement of $R_{e}$. The general expectation is that its value $R_{e, \text { ph }}$ should be slightly above $R_{c, \text { ph }}$, e.g., $0.8 \mathrm{fm} \leq R_{e, \mathrm{ph}} \leq 1.0 \mathrm{fm}$, but it is not clear which precise value to choose. Certainly, there exist further observables impeding very concentrated energy densities, because the physical expectation is that the energy density is rather flat in the core of a nucleus (nucleon), with a pion tail close to the surface. In addition, the results of section 5.2 show that the energy densities may be flattened easily, without affecting the Roper resonances.

Further physical restrictions on Skyrme model parameters are obviously provided by properties of nuclei, corresponding to Skyrmions with baryon number $B>1$. Their treatment, however, requires full three-dimensional numerical calculations. Any reduction of the parameter space which can be achieved in the $B=1$ sector alone is, therefore, very important because it reduces the need for these full three-dimensional numerical calculations.

To summarize, our main result is that generalised versions of the Skyrme model are perfectly capable of reproducing the Roper resonances and other observables with high precision. The further investigation of these generalised Skyrme models is, therefore, a promising and very timely research direction in the quest for a reliable low-energy effective field theory of strong interactions and nuclear physics. 


\section{Acknowledgments}

C.A. and A.W. acknowledge financial support from the Ministry of Education, Culture, and Sports, Spain (Grant No. FPA 2014-58-293-C2-1-P), the Xunta de Galicia (Grant No. INCITE09.296.035PR and Conselleria de Educacion), the Spanish Consolider-Ingenio 2010 Programme CPAN (CSD2007-00042), Maria de Maetzu Unit of Excellence MDM-20160692, and FEDER. Some of the work of M.H. was undertaken at the Department of Mathematics and Statistics, University of Massachusetts, financially supported by FP7, Marie Curie Actions, People, International Research Staff Exchange Scheme (IRSES-606096).

Open Access. This article is distributed under the terms of the Creative Commons Attribution License (CC-BY 4.0), which permits any use, distribution and reproduction in any medium, provided the original author(s) and source are credited.

\section{References}

[1] T.H.R. Skyrme, A nonlinear field theory, Proc. Roy. Soc. Lond. A 260 (1961) 127 [InSPIRE].

[2] T.H.R. Skyrme, A unified field theory of mesons and baryons, Nucl. Phys. 31 (1962) 556.

[3] T.H.R. Skyrme, Kinks and the Dirac equation, J. Math. Phys. 12 (1971) 1735 [InSPIRE].

[4] G. 't Hooft, A Planar Diagram Theory for Strong Interactions, Nucl. Phys. B 72 (1974) 461 [INSPIRE].

[5] E. Witten, Baryons in the 1/n Expansion, Nucl. Phys. B 160 (1979) 57 [INSPIRE].

[6] E. Witten, Current Algebra, Baryons and Quark Confinement, Nucl. Phys. B 223 (1983) 433 [INSPIRE].

[7] G.S. Adkins, C.R. Nappi and E. Witten, Static Properties of Nucleons in the Skyrme Model, Nucl. Phys. B 228 (1983) 552 [inSPIRE].

[8] G.S. Adkins and C.R. Nappi, The Skyrme Model with Pion Masses, Nucl. Phys. B 233 (1984) 109 [INSPIRE].

[9] A. Jackson, A.D. Jackson, A.S. Goldhaber, G.E. Brown and L.C. Castillejo, A Modified Skyrmion, Phys. Lett. B 154 (1985) 101 [INSPIRE].

[10] G. Holzwarth and B. Schwesinger, Baryons in the Skyrme Model, Rept. Prog. Phys. 49 (1986) 825 [INSPIRE].

[11] I. Zahed and G.E. Brown, The Skyrme Model, Phys. Rept. 142 (1986) 1 [INSPIRE].

[12] B. Schwesinger and H. Weigel, Vector Mesons Versus Higher Order Terms in the Skyrme Model Approach to Baryon Resonances, Nucl. Phys. A 465 (1987) 733 [InSPIRE].

[13] H. Weigel, B. Schwesinger and G. Holzwarth, Exotic Baryon Number B $=2$ States in the SU(2) Skyrme Model, Phys. Lett. B 168 (1986) 321 [INSPIRE].

[14] B. Schwesinger, H. Weigel, G. Holzwarth and A. Hayashi, The Skyrme Soliton in Pion, Vector and Scalar Meson Fields: $\pi N$ Scattering and Photoproduction, Phys. Rept. 173 (1989) 173 [INSPIRE].

[15] E. Braaten and L. Carson, The Deuteron as a Soliton in the Skyrme Model, Phys. Rev. Lett. 56 (1986) 1897 [INSPIRE]. 
[16] E. Braaten and L. Carson, The Deuteron as a Toroidal Skyrmion, Phys. Rev. D 38 (1988) 3525 [INSPIRE].

[17] L. Carson, B = 3 nuclei as quantized multiskyrmions, Phys. Rev. Lett. 66 (1991) 1406 [INSPIRE].

[18] T.S. Walhout, Multiskyrmions as nuclei, Nucl. Phys. A 531 (1991) 596 [INSPIRE].

[19] O.V. Manko, N.S. Manton and S.W. Wood, Light nuclei as quantized skyrmions, Phys. Rev. C 76 (2007) 055203 [arXiv:0707.0868] [INSPIRE].

[20] R.A. Battye, N.S. Manton, P.M. Sutcliffe and S.W. Wood, Light Nuclei of Even Mass Number in the Skyrme Model, Phys. Rev. C 80 (2009) 034323 [arXiv:0905.0099] [InSPIRE].

[21] C.J. Halcrow, Vibrational quantisation of the B $=7$ Skyrmion, Nucl. Phys. B 904 (2016) 106 [arXiv: 1511.00682] [INSPIRE].

[22] P.H.C. Lau and N.S. Manton, States of Carbon-12 in the Skyrme Model, Phys. Rev. Lett. 113 (2014) 232503 [arXiv: 1408.6680] [INSPIRE].

[23] C.J. Halcrow, C. King and N.S. Manton, A dynamical $\alpha$-cluster model of ${ }^{16} O$, Phys. Rev. C 95 (2017) 031303 [arXiv: 1608.05048] [INSPIRE].

[24] C. Adam, C. Naya, J. Sanchez-Guillen and A. Wereszczynski, Bogomol'nyi-Prasad-Sommerfield Skyrme Model and Nuclear Binding Energies, Phys. Rev. Lett. 111 (2013) 232501 [arXiv: 1312.2960] [INSPIRE].

[25] C. Adam, C. Naya, J. Sanchez-Guillen and A. Wereszczynski, Nuclear binding energies from a Bogomol'nyi-Prasad-Sommerfield Skyrme model, Phys. Rev. C 88 (2013) 054313 [arXiv: 1309.0820] [INSPIRE].

[26] M. Gillard, D. Harland and M. Speight, Skyrmions with low binding energies, Nucl. Phys. B 895 (2015) 272 [arXiv:1501.05455] [INSPIRE].

[27] M. Gillard, D. Harland, E. Kirk, B. Maybee and M. Speight, A point particle model of lightly bound skyrmions, Nucl. Phys. B 917 (2017) 286 [arXiv:1612.05481] [INSPIRE].

[28] S.B. Gudnason, Loosening up the Skyrme model, Phys. Rev. D 93 (2016) 065048 [arXiv: 1601.05024] [INSPIRE].

[29] S.B. Gudnason, B. Zhang and N. Ma, Generalized Skyrme model with the loosely bound potential, Phys. Rev. D 94 (2016) 125004 [arXiv:1609.01591] [InSPIRE].

[30] S.B. Gudnason and M. Nitta, Modifying the pion mass in the loosely bound Skyrme model, Phys. Rev. D 94 (2016) 065018 [arXiv:1606.02981] [INSPIRE].

[31] C. Adam, C. Naya, J. Sanchez-Guillen, R. Vazquez and A. Wereszczynski, BPS Skyrmions as neutron stars, Phys. Lett. B 742 (2015) 136 [arXiv:1407.3799] [INSPIRE].

[32] C. Adam, C. Naya, J. Sanchez-Guillen, R. Vazquez and A. Wereszczynski, Neutron stars in the Bogomol'nyi-Prasad-Sommerfield Skyrme model: Mean-field limit versus full field theory, Phys. Rev. C 92 (2015) 025802 [arXiv: 1503.03095] [INSPIRE].

[33] S.G. Nelmes and B.M.A.G. Piette, Skyrmion stars and the multilayered rational map ansatz, Phys. Rev. D 84 (2011) 085017.

[34] S.G. Nelmes and B.M.A.G. Piette, Phase transition and anisotropic deformations of neutron star matter, Phys. Rev. D 85 (2012) 123004. 
[35] Particle Data Group collaboration, K.A. Olive et al., Review of Particle Physics, Chin. Phys. C 38 (2014) 090001 [inSPIRE].

[36] P. Dorey and T. Romanczukiewicz, Resonant kink-antikink scattering through quasinormal modes, Phys. Lett. B 779 (2018) 117 [arXiv:1712.10235] [INSPIRE].

[37] C. Adam, M. Haberichter and A. Wereszczynski, Skyrme models and nuclear matter equation of state, Phys. Rev. C 92 (2015) 055807 [arXiv: 1509.04795] [InSPIRE].

[38] C. Adam, C. Naya, J. Sanchez-Guillen, J.M. Speight and A. Wereszczynski, Thermodynamics of the BPS Skyrme model, Phys. Rev. D 90 (2014) 045003 [arXiv:1405.2927] [INSPIRE].

[39] L.P. Teo, Fermionic Casimir interaction in cylinder-plate and cylinder-cylinder geometries, Phys. Rev. D 91 (2015) 125030 [arXiv:1504.05317] [INSPIRE].

[40] A. Jackson, A.D. Jackson, A.S. Goldhaber, G.E. Brown and L.C. Castillejo, A Modified Skyrmion, Phys. Lett. B 154 (1985) 101 [INSPIRE].

[41] C. Hajduk and B. Schwesinger, The breathing mode of nucleons and $\Delta$-isobars in the Skyrme model, Phys. Lett. B 140 (1984) 172 [INSPIRE].

[42] U.B. Kaulfuss and U.G. Meissner, The Breathing Mode of the Modified Skyrmion, Phys. Lett. B 154 (1985) 193 [INSPIRE].

[43] A. Hayashi and G. Holzwarth, Excited nucleon states in the Skyrme model, Phys. Lett. B 140 (1984) 175 [INSPIRE].

[44] L.C. Biedenharn, Y. Dothan and M. Tarlini, Rotational-Vibrational Coupling in the Skyrmion Model for Baryons, Phys. Rev. D 31 (1985) 649 [INSPIRE].

[45] C. Adam, C. Naya, J. Sanchez-Guillen and A. Wereszczynski, Rotational-vibrational coupling in the BPS Skyrme model of baryons, Phys. Lett. B 726 (2013) 892.

[46] I. Zahed, U.G. Meissner and U.B. Kaulfuss, Low lying resonances in the Skyrme model using the semiclassical approximation, Nucl. Phys. A 426 (1984) 525 [INSPIRE].

[47] J.D. Breit and C.R. Nappi, Phase Shifts of the Skyrmion Breathing Mode, Phys. Rev. Lett. 53 (1984) 889 [INSPIRE].

[48] W.T. Lin and B. Piette, Skyrmion Vibration Modes within the Rational Map Ansatz, Phys. Rev. D 77 (2008) 125028 [arXiv:0804.4786] [INSPIRE].

[49] P. Bizon, T. Chmaj and A. Rostworowski, On asymptotic stability of the Skyrmion, Phys. Rev. D 75 (2007) 121702 [math-ph/0701037] [INSPIRE].

[50] C. Adam, M. Haberichter, T. Romanczukiewicz and A. Wereszczynski, Radial vibrations of BPS skyrmions, Phys. Rev. D 94 (2016) 096013 [arXiv: 1607.04286] [INSPIRE].

[51] M. Heusler, S. Droz and N. Straumann, Stability analysis of selfgravitating skyrmions, Phys. Lett. B 271 (1991) 61 [INSPIRE].

[52] R.A. Battye and P.M. Sutcliffe, Skyrmions, fullerenes and rational maps, Rev. Math. Phys. 14 (2002) 29 [hep-th/0103026] [InSPIRE].

[53] U. Ascher, J. Christiansen and R. Russell, Collocation Software for Boundary-Value ODEs, ACM TOMS 7 (1981) 209.

[54] U. Ascher, J. Christiansen and R. Russell, A collocation solver for mixed order systems of boundary value problems, Math. Comput. 33 (1979) 659 [INSPIRE].

[55] Scipy, scientific tools for python, version 0.6.0, http://www.scipy.org. 
[56] T. Ioannidou and A. Lukacs, Time-dependent Bogomolny-Prasad-Sommerfeld skyrmions, J. Math. Phys. 57 (2016) 022901 [arXiv:1601.03048] [InSPIRE].

[57] P. Dorey, A. Halavanau, J. Mercer, T. Romanczukiewicz and Y. Shnir, Boundary scattering in the $\phi^{4}$ model, JHEP 05 (2017) 107 [arXiv: 1508.02329] [INSPIRE].

[58] T. Romanczukiewicz and Y. Shnir, Oscillons in the presence of external potential, JHEP 01 (2018) 101 [arXiv: 1706. 09234] [INSPIRE].

[59] C. Adam, J. Sanchez-Guillen and A. Wereszczynski, A Skyrme-type proposal for baryonic matter, Phys. Lett. B 691 (2010) 105 [arXiv:1001.4544] [INSPIRE].

[60] C. Adam, J. Sanchez-Guillen and A. Wereszczynski, A BPS Skyrme model and baryons at large $N_{c}$, Phys. Rev. D 82 (2010) 085015 [arXiv: 1007.1567] [InSPIRE]. 